Božo Vodušek

CDU $803-559.13$

\title{
ÜBER NICHTONOMATOPOETISCHE IKONISCHE SYNONYMIE
}

ilan ist daran gewohnt, die Darstellung gegebener lexikalischer Sinngehalte in verschiedenen Sprachen durch verschiedene Laute festzustellen und daraus ein allgemeines Prinzip der Willkürlichkeit sprachlicher Zeichen abzuleiten, die Tatsache jedoch, dass wir neben der Lautverschiedenheit bei interlingualen und auch intralingualen Synonymen häufige Lautgleichheit konstatieren können, wird in der linguistischen Theorie regelmässig ausser Acht gelassen. Diese Tatsache zeigt nun offenbar auf sprachlichen Ikonismus, und die Lösung des Widerspruchs ist eine eminente Aufgabe der Semiotik die sich eigens mit der Analyse der Zeichenprozesse beschäfigt.

In teils veröffentlichten, teils noch unveröffentlichten studien habe ich eine ausfihrliche Untersuchung der lautlichen Motiviertheit in Synonymenreihen aus verschiedenen indoeuropäischSprachen durchgefuhrt, und zwar am Buch von C.D.Buck: A Dictionary of Selected Synonyms in the Principal Indo-European Languages. Die Hauptresultate dieser Untersuchungen samt einem Versuch ihrer semiotischen Deutung möchte ich in diesem Vortrag vorlegen.

Da jedoch die Resultate der Untersuchungen zu einer weitreichenden Revision der traditionellen negativen Stellungnahme zum Problem des sprachlichen Ikonismus fuhren, ist es wohl angebracht, sich vorerst die theoretische Begriundung dieser stellungnahme, die unserer Deutung widerspricht, näher anzusehen. Wir wählen dazu am besten die Begrundung, die vom bekanntesten und einflussreichsten Vertreter der Lehre von der Willkülichkeit sprachlicher Zeichen, F.de Saussure, in seinem Cours de linguistique générale dargelegt worden ist. Grundsätzlich wird zwar von 
de Saussure die Existenz des Ikonismus in der Sprache zugestanden, aber dieses Zugeständnis wird sogleich durch zwei wesentliche Einschränkungen entkräftet. Erstens werden als möglicher Fall des sprachlichen Ikonismus nur Onomatopöien, d.h. Nachahmungen aussersprachlicher Laute, und in enger Verwandschaft mit ihnen sogenannte primäre Interjektionen angeführt, wobei einerseits diachronisch die Zerstörung ihrer lautlichen Motiviertheit durch den Iautwandel, andererseits synchronisch ihr approximativer und konventionell bedingter Charakter hervorgehoben wird. Zweitens wird ausdrucklich die geringe Anzahl der Onomatopöe betont, die weit geringer sein soll, als gewöhnlich angenommen werde, woraus ihre Bedeutungslosigkeit klar hervorgehe. Das sind die Argumente, auf Grund derer es de Saussure als bewiesen betrachtet, dass die ikonischen Symbole uberhaupt keine organischer Elemente der Sprache darstellen, sonderm ausserhalb des sprachsystems zu stellen sind.

Es ist nicht ohne Bedeutung darauf hinzuweisen, dass diese Stellungnahme keineswegs als eine originelle Erkenntnis de Saussures zu bewerten ist; sie geht direkt aus der gleichen Anschauung der Junggrammatiker hervor, die der allgemeinen positivistischen Einstellung des letzten Viertels des vorigen Jahrhunderts entsprach. Das besondere Gewicht der Stellungnahme de Saussures besteht jedoch darin, dass sie den Ausgangspunkt des strukturalismus in der Iinguistik bedeutet. Die erwiesenen Erfolge der neuen Forschungsmethode im Zusammenwirken mit einer allgemein akzeptierten Tradition wandten eine lange Zeit die Aufmerksamkeit der Forscher von der systematischen Prüfung der Argumente de Saussures ab. So können wir heute - im Rahmen der mehrtausendjährigen Diskussion seit Plato's Kratylos, ob die sprache vorwiegend physei oder thesei determiniert sei auf eine hundertjährige ununterbrochene Periode der absoluten Vorherrschaft der thesei-Hypothese zuruckblicken. Während dieser ganzen Zeit wurden die Versuche, ein inneres Band zwischen dem sprachlichen signans und dem aussersprachlichen signatum ausserhalb der onomatopöien im engsten Sinne aufzudecken, systematisch 
abgelehnt, obgleich sie nur vom synchronistischen Standpunkt aus operierten. Im Zusammenhang damit das Problem des Ursprungs der sprache aufzuwerfen, wurde um so schärfer als Dilettantismus gebrandmarkt: nach de Saussures extremer, aber die allgemeine Überzeugung spiegelnder Formulierung solite es sich nicht lohnen, die Frage danach uberhaupt zu stellen. Bezeichnend fur die Atmosphäre, in der nur die Hypothese von der Willkirlichkeit der Sprachzeichen als wissenschaftlich begrindet angesehen wurde, ist der Fall solcher Forscher wie M.Grammont und J.R. Firth, welche die "impressive" oder "phonästhetische" Funktion der Sprachlaute klar erkannten und in ihren sprachtheoretischen Betrachtungen ausdrlicklich hervorhoben, diese Funktion als eine subjektive - wenn auch kollektive - Erscheinung bezeichneten, ohne den offenbaren Widerspruch zwischen "subjektiv" und "kollektiv" zu beachten. Ähnlich blieben die Untersuchungen von A. Meillet's Pariser Schule uber die "Expressivität" der Laute und Lautverdoppelungen, sowie die parallelen, von der Dialektologie und dem etymologischen Studium herkommende Forschungen, z.B. die yon K.Jaberg und V.Machek, auf einem blinden Nebengeleise stecken, weil sie sich mit de Saussures Prinzip der Willkirlichkeit der sprachlichen Zeichen nicht auseinandersetzen wollten oder wussten.

Nun ist die Beweisfihrung de Saussures zugunsten dieses Prinzips gar nicht stichhaltig, denn der Schluss, dass die Onomatopöien und andere ikonische Sprachsymbole ausserhalb des sprachsystems zu stellen sind, beruht im Grunde auf einem durchaus subjektiven Urteil uber ein sprachliches Quantitätproblem. Nur, wenn die Zahl der ikonischen Sprachzeichen wirklich so unbedeutend wäre wie es Saussure von vornherein annimmt, könnte ihre ausdrickliche Ausklammerung bei der Aufstellung der Grundprinzipien der allgemeinen sprachtheorie irgendwie berechtigt erscheinen. Einen realen quantitativen Beweis dafur ist jedoch Saussure schuldig geblieben und die mangelnde Bestäigung dieser so wichtigen Annahme in seiner sprachtheorie ist bis heute nicht ersetzt 
worden. Vom heutigen Stand der Linguistik her gesehen ist es indessen klar, dass die Kontroverse über das Bestehen und das Ausmass lautlich motivierter: Zeichen innerhalb eines Sprachsystems eine quantitative, d.h. statistische Iösung verlangt. Einer solchen, statistischen Behandlung ist das indoeuropäische Sprachmaterial, das in Buck's Wörterbuch enthalten ist, von mir unterzogen worden. Im Rahmen dieser Untersuchung hat es sich herausgestellt, dass hier den konventionellen Sprachzeichen als Gegenpol nicht nur ein paar onomatopöien und primäre Interjektionen entgegestehen, sondern eine ansehnliche Zahl onomatopoetischer und nichtonomatopoetischer Sprachzeichen, deren Unkonventionalität durch den Synonymentest objektiv festgestelit werden konnte. Auf der Grundlage einer von jeder Bevorzugung freien, gleichmässig auf das ganze Buch verteilten Beispielssammlung von 110 Synonymenreihen wurde die Phonempopulation der indceuropäischen Wurzelmorpheme der im wörterbuche enthaltenen Synonymenmasse errechnet und mit dem Phonembestand einzelner Synonymenreihen und begriffsverwandter Gruppen von Synonymenreihen verglichen, was uberraschende Ergebnisse zu Tage förderte.

Ein paar nähere Angaben uber die angewandte statistische Methode, die zum Verständnis der darzustellenden Resultate notwendig sind, seien kurz angefuhrt. Da die Konventionalität oder Unkonventionalität von Synonymen sich in der wiederholung oder Nichtwiederholung der gleichen Lautelemente äussert und der wiederholungsprozess nur auf der Ebene eines identischen Phonemsystems verlasslich kontrolliert werden kann, mussten alle behandelten synonyme grundsätzlich auf die rekonstruierte indoeuropäische Form ihrer lexikalischen Kemelemente zuruckgefuhrt werden. In jeder Synonymenreihe wurden nur etymologisch unverwandte synonyme statistisch bericksichtigt; fur verwandte synonyme wurde nur ein einzelnes Wurzelmorphem als gemeinsamer Ausgangspunkt in Betracht gezogen. Dazu wurde, um das Postulat der Unabhängigkeit statistischer Einheiten vol zu sichern, ưberall auf unabgeleitete Wurzelmorpheme zurükgegriffen, die etymologisch weiterhin nicht analysierbar sind. Die in den Wurzelmorphemen auftretenden 
Phoneme wurden bei der Zählung in 11 klassen eingeteilt: war es möglich bei der Berechnung der Phonempopulation einen genügenden Umfang der einzelnen Phoremklassen zu erreichen, si richtig gegeneinander abzuwägen und gleichzeitig einer Zersplitterung der Resultate vorzubeugen. Als ronsonantenklassen wurden $L$ und $R$, jedes Phonem fur sich, selbstständig gezählt, und neben ihnen die zusammengesetzten lategorien der Vasale /N/, Labiale /P/, Dentale /T/, Velare / $/ \mathrm{d}$ und Sibilanten/S/. Der artikulatorischen Einteilung der Konsonanten entsprach in gleicher Form artikulatorische Distribution der Vokale: U samt den U-haltigen fallenden Diphthongen, I samt den I-haltigen fallenden Diphthongen, dann A als das dritte Glied des vokalischen Dreiecks und die neutrale $\mathrm{E} / \mathrm{O}-\mathrm{K}$ lasse. Die Wurzelmorpheme mit steigenden Diphthongen, enthaltend $I$ und $U$, wurden ausgeschieden und einer eigener Zahlung unterworfen. Die verschiedene Behandlung der fallenden und steigenden Diphthonge entstand im Hinblick auf das Strukturprinzip der indoeuropäischen Wurzelmorpheme, wonach in ihnen wegen des Ablaut fallende Diphthonge mit $U$ und $I a b-$ wechseln, was hingegen bei steigenden Diphthongen nicht der Fall ist. Ein zweifaches Auftreten der gleichen Phonemkategorie in demselben Wurzelmorphem, das sich etwas häufiger, nur bei den asalen vorfand, wurde als einfach gezählt, denn, was zu ermitteln war, sollte keine Sammlung der Phoneme an sich, sondern eine Sammlung der Phonemcharakteristiken der Wurzelmorpheme sein, und die Reduplikation, welche zwar als besondere Art der Lautmotivierung aufgefasst werden muss, befindet sich innerhalb des gleichen Morphems. Aus der beschriebenen Berechnung der Phonempopulation, der eine gleichartige Berechnung des Phonembestandes jeder beliebigen Synonymenreihe entsprach, mussten freilich jene Synonyme ausgeschlossen bleiben, die einer etymologischen Erklärung ermangeln sowie diejenigen ausserindoeuropäischer Herkunft, und einzelsprachliche, als imitativ bezeichnete synonyme, welche jedoch zusammen kaum 14\% der festgesteliten unverwandten Wörter im repräsentativen Muster der 110 synonymenreihen darstellen. So wurde eine breite, verlässliche Basis fur die Bestimmung der durchschnittlichen Frequenz für jede Phonemkategorie 
geschaffen, zusammen mit der Möglichkeit, für beliebige, genug grosse Gruppen von Synonymenreihen die ungewöhnliche wiederholung einer Phonemkategorie in den lexikalischen Kermelementen der Synonyme auf inre statistische Signifikanz hin zu testen.

Der Test, der von mir ausgefuhrt worden ist,erstreckte sich auf alle aufgezahlten Phonemkategorien mit Ausnahme der Kategorie der steigenden Diphthonge wegen ihrer schwierigen Einordnung in das aufgestellte Modell der Phonemverteilung. Zum Zweck möglichst gleicher Behandlung der auftretenden Wurzelmorpheme und der damit verbundenen Geometrisierung des Modells wurden beim Testen ausser den Wurzelmorphemen mit steigenden Diphthongen auch die dritten und die vierten Konsonanten und die sehr seltenen zweiten Vokale der Wurzelmorpheme unberucksichtigt gelassen, was jedoch zu keiner beachtlichen Verschiebung des gegenseitigen Verhalltnisses der Phonemkategorien fuhrte und nur kaum $12 \%$ der Phonemmasse bei Vokalen bzw. kaum 16\% bei Konsonanten betraf. Ich muss das hier erwähnen, weil der publizierte Test, der sich auf die Konsonantenkategorien bezieht, unter dieser unschädlichen, wegen grösserer Anschaulichkeit und Verlässlichkeit sogar vorteilhaften Beschräkung durchgefuhrt wurde /Iinguistica, VI-X, 1964-1970, Ijubljana/. Die Gruppen von Synonymenreihen, die mit dem repräsentativen Muster der 110 Serien nacheinander verglichen wurden, betrugen je ungefähr 30 serien oder auch mehr und waren auf Grund ihrer vermutlichen semantischen Verwandtschaft zusammengestellt worden. Die Ergebnisse der Vergleiche lieferten - mit der Wahrscheinlichkeit einer zufälligen Anhaufung von charakteristischen Phonemelementen in der Höhe von höchstens einigen Tausendsteln zu eins - den strikten Beweis, dass zwischen Iaut und Sinn im weiten Rahmen von Hunderten ausgewahlter serien aus Bucks Wörterbuch ein inneres Band anzunehmen ist. $\mathrm{Da}$ das wörterbuch alle Bereiche des menschlichen Lebens und die wichtigsten Erscheinungen der äusseren welt zu erfassen versucht, jedoch keine spezielle Sammlung der Ausdrücke fur Tiemamen und tierische Lautäusserungen enthält, war es von Anfang an unmöglich, eine grössere Anzahl von Synonymenreihen 
zusammenzubringen, die bei den angeführten synonymen auf eine bestimmte onomatopoetische Verbindung zwischen ihrer lautlichen Form und den bezeichneten aussersprachlichen Phänomenen schliessen machen könnte. Die den Vergleichen unterworfenen Serien von synonymen Ausdrücken bezogen sich grösstenteils auf unhörbare Erscheinungen, und wenn die Existenz eines inneren Bandes zwischen Laut und Sinn in diesen Fallen zu Tage trat, musste notwendigerweise dieses Band in der uberwiegenden Mehrzahl nichtonomatopoetischer Natur sein.

Es könnten vollständige Listen der im Test inbegriffenen Serien samt den dazugehörigen Zahlen zux Bekrłftigung obiger Feststellungen angefuhrt werden, doch wirde das ohne einen speziellen Kommentar für jede Serie, was an dieser stelle unmöglich ist, keine richtige Information bieten. Wir wollen uns deshalb gleich der Beschreibung und Analyse einiger typischen Serien mit uberdurchschnittlicher Frequenz bestimnter Phonemkategorien zuwenden; an ihnen werden wir am besten den Ikonismus, um den es geht, beurteilen können. Obgleich numerische Daten, wenn sie sich im kleinen Rahmen bewegen, keine zwingenden Schlusse uber die Zufälligkeit oder Unzufälligkeit der synonymischen Phonemwiederholungen zulassen, sind sie in Verbindung mit einer allgemeinen strukturellen Analyse der betreffenden Serie sehr aufschlussreich. Zur Einleitung - um vom Bekannten zum Unbekannten fortzuschreiten - wählen wir ausnahmsweise eine in der Minderheit sich befindenden onomatopoetisch bedingten serien: es ist das die Serie SPEAK, TALK unter der fortlaufenden Nummer 18.21 in Bucks wörterbuch /= B 18.21/. Sie enthät einschliesslich zweier 2 Synonymen, die auf U్n-Morpheme zuruckgehen, 23 etymologisch erklärte und 7 unerklärte synonyme, denen sich noch ein einzelsprachliches Synonym zugesellt: /Ling. VII/I, 41 f./. Unter den 23 erschlossenen Wurzelmorphemen zu den einzelnen Ausdricken treten 9 Morpheme mit der I-Charakteristik auf, und zwar 1eg'zu Gr. Iegō, lā- zu Gr. laleó, tolk ${ }^{\mathrm{W}}=\mathrm{zu}$ Lat. loquī, $\mathrm{g}^{\mathrm{W}} \mathrm{el}-$ uber Gr. parabolèe, Vlat. paraboläre zu It. parlare, plab- zu Ir. labrur, del- zu An. tala, kel- zu lit. kalbeti, gal- zu KSlav. glagolati, und bhel- zu Alit. bilti usw. Diese 9 Morpheme sind 
dreimal mehr als der Durchschnitt, der im Rahmen von 23 Einheiten kaum 3 I-Morpheme beträgt. Nun ist es interessant zu konstatieren, dass von den 9 angefuhrten Morphemen 5 nach einheitlicher Meinung der Etymologen auf Schallnachahmung beruhen: $1 \bar{a}-$, plab-, kēl-, gal-, bhel-. Die Vermutung vom Vorhandensein onomatopoetischer Ikonen, folgend aus der quantitativen Phonemanalyse, und die traditionelle etymologische Stellungnahme stimmen also vollkommen uberein. Um den zugrunde liegenden Zeichenprozess deuten zu können, durfen wir uns jedoch nicht mit dieser prinzipiellen Feststellung begnugen; wir mussen die Struktur der onomatopoetischen Ikonen durch ihren gegenseitigen Vergleich năher bestimmen. Es zeigt sich, dass das $L$ in allen Schallnachahmungen als zweiter Konsonant aufritt, ausser in dem reduplizierten wurzelmorphem 1̄a-. Es zeigt sich aber daruber hinaus, dass sogar alle anderen, als konventionelle sprachzeichen betrachtete Wurzelmorpheme mit I, mit der einzigen Ausnahme von leg- in Gr. legō, die gleiche Struktur aufweisen. In den Irilorphemen wird also durchgehends das $\mathrm{I}$ an zweiter Konsonantenstelle bevorzugt. Ist das ein Zufall? Wenn wir bei einer weiteren quantitativen Analyse die Sache generell anfassen und alle Lautkombinationen vom Typus: Konsonant + I gleichstellen, bekommen wir keine Antwort, da ca. drei viertel der I-Morpheme, von denen unsere Phonempopulation abgeleitet ist, durch dieselbe Struktur gekennzeichnet sind. Wir wollen deshalb die Frage offen lassen und nur darauf aufmerksam machen, dass auch die zwei etymologisch nicht geklärten Synonyme: Ngr. milö und Čech. mluviti von der festgestellten Jautformel nicht abweichen. Indessen muss noch eine andere Frage besprochen werden. Die Statistik, die wir betreiben, bewegt sich auf einer panchronischen Ebene, denn obgleich alle Synonyme auf ihr indoeuropäisches Ursprungsmorphem zurikckgefuhrt worden sind, gehören sie doch verschiedenen historischen Phasen der einzelnen Glieder der indoeuropäischen sprachfamilie an. Wie sieht nun das behandelte Lautikonenproblem in diachronischer Perspektive aus? Von den 9 I-Morphemen der Liste haben ihre Ableger in den lebenden indoeuropäischen sprachen nur 4, nämlich 2 ikonische und 2 als 
konventionell betrachtete Wurzelmorpheme. Vom Morphem plababgeleitet sind NIr. Iabhraim und Cym. llefaru, von kel- lit. kalbèti, von del- Dän. tale und Schw. tala nebst ile. talk, von $g^{W}$ el-It. parlare, Fr. parler. Die Synonyme, die auf anderen I-Worphemen beruhten, sind im Laufe der Zeiten verschwinden. Was bei diesem Wortpanorama besonders beachtenswert ist, erkennt man jedoch an der parallelen Ausmerzung jeglichen Unterschieds im Bewusstsein der modernen Sprecher zwischen Synonymen onomatopoetischen Ursprungs und zwischen den anderen; die angefuhrten modernen Ausdrücke für "sprechen" mit einer L-Charakteristik werden gewiss alle als unonomatopoetisch empfunden. Angesichts dieser Tatsache ist man versucht, die sprachentwicklung in der einseitigen Richtung des Verschwindens bzw. der Entwertung des Ikonismus zu betrachten. Dementgegen besitzen wir gerade in der Geschichte des italienischen Synonyms: parlare den Hinweis auf ein komplementäres Geschehen in entgegengesetzter Richtung. Dass Lat. loqui durch ein von dem Griechischen entlehnten parabolè abgeleitetes Vlat. parabolāre ersetzt wurde, mag mehrere Grinde haben. Es ist aber sicher aufallig, dass man dabei wieder auf die I-Charakteristik in der bekannten stellung stösst, die genau an das onomatopoetische wurzelmoxphem plab- erinnert, freilich mit dem gleichzeitigen Auftreten eines $\mathrm{R}$ in Präfix, das jedoch wieder seine Parallele im romanischen imitativen Wurzelmorphem čar-, vertreten durch das Cym. siarad, besitzt. Es muss bedacht werden, dass das Lat. loqui wegen des Verlustes seiner urspringlichen Lautcharakteristik mit $I$ an zweiter Konsonantenstelle sich, ungeachtet der Möglichkeit seiner ikonischen Herkunft für eine onomatopoetische Interpretation gar nicht eignete; daher ist es sehr wahrscheinlich, dass die Wahl von: parabolāré für "sprechen" im vulgären, familiarren sprachgebrauch in erster Linie durch das Streben nach einem lautlich motivierten Kraftausdruck bedingt wurde. Dirrfen wir nicht in demselben Bedirfnis den Ausgangspunkt einer anderen romanischen Ersetzung fir: loqui sehen, nämlich des Sp. hablar aus: fābulāre? Die Lautform ist wesentich die gleiche: zwei Labiale und dann L; die Annahme des 
subjektiven Gefihls der lautlichen Motiviertheit wird hier ausserdem durch die klar überlieferte Bedeutung des: fābulāre bzw. fābulārī "plauderm", "schwatzen" und den verächtlichen Nebengebrauch des Grundwortes: fābul "dummes Zeug" gestutzt. Heute ist bei diesen sekundären Onomatopöien ein solches subjektives Gefuhl zwar ebenso tot wie bei denen aus einer älteren Zeit, die als primär gelten; doch die uberraschende Übereinstimmung der Lautform in beiden Fälen mit der alten onomatopoetischen Lautformel bildet ein objektives Kriterium fur die Feststellung ihrer einstigen Rolle onomatopoetischer Ikonen.

Wenden wir uns jetzt einer anderen Serie mit einer bestimmten uberdurchschnittlichen Lautcharakteristik zu; diesmal beziehen sich die Synonyme auf ein unhörbares Phänomen und die Lautcharakteristik ist vokalisch. Es handelt sich um die serie BRIGHT /B 15.57; Iing. VII/l, 32 f./. Die 21 bekannten primären Wurzelmorpheme der Serie - 2 Synonyme sind unerklärt und 1 gilt als einzelsprachlich imitativ - weisen 6mal eine I-charakteristik auf, während der Durchschnitt nur 3 bzw. höchstens 4, je nachdem man das Wurzelmorphem i $\bar{a} \bar{a}-$ mit steigendem I-Diphthong bei der Zahlung mit einbezieht oder nicht, zulassen wirde. Das sind $\mathrm{g}^{\mathrm{W} h \bar{e} i-} \mathrm{zu}$ Gr. phaidros, nei- zu Lat. nitidus, sk'āi- zu An. skāērr, aisk-bzw. ai-zu Skr. jasan, dei- zu Ai. dyumantund $\mathrm{kthe} / \mathrm{i} / \mathrm{zu}$ Av. xšäta-. Um diesen Umstand besser beurteilen zu können, heben wir hervor, dass dementgegen die drei auftretenden U-Charakteristiken und die 3 bzw. 4 A-Charakteristiken sich unter dem Durchschnitt /U/ oder nicht dariber befinden /A/. Aber das tberwiegen der I-Charakteristik tritt noch viel stärker an den Tag, wenn wir uns die vokalischen Erweiterungen bzw. Determinative - die morphologisch unerklärt sind ansehen. Im Synonym Cym. disglair haben wir zwar mit einer Grundform k'el- mit neutralem E-Vokalismus zu tun, der jedoch in der faktischen Erweiterung $\mathrm{K}^{\prime} l e i$ - einer I-Charakteristik weicht. Neben Nir. geal, berwhend auf g'hel-, haben wir in derselben Sprache das Synonym glēineach, das auf g'hlei- zurückgeht. Und das Wurzelmorphem k'eu-, welches als Grundform des Iit. šviesus 
und des Kslav. svĕtĭlŭ erwiesen ist, tritt wiederum in beiden nur in der erweiterten Form $\mathrm{K}^{\prime}$ wei- auf. In diesem letzten Fall kann man geradezu von einer Umformung einer negativen U-Charakteristik in eine positive I-Charakteristik sprechen. Da im Durschnitt eine solche Erweiterung kaum auf jede zweite Serie entfallt, ist an einer besonderen Ursache fur ihr wiederholtes Auftauchen in unserer Synonymenreihe schwer zu zweifeln. Die uberzähligen I-Diphthonge als Wurzeldeterminative stellen eine genaue Parallele der uberdurchschnittlichen I-Diphthonge in den eigentlichen Wurzelmorphemen dar und verkleinern die Möglichkeit eines Zufalls auch fur jene. In diesem Zusammenhang fällt noch die Anwesenheit des schon erwähnten Wurzelmorphems īazum russischen Synonym jarkij in die Augen, da Wurzelmorpheme mit î im steigenden Diphthong nur 25mal im repräsentativen Muster der 110 Synonymenreihen erscheinen. Ohne sich in etymologische Spekulationen einzulassen, genügt es, darauf aufmerksam zu machen, dass in mehreren Beispielen die Ableitung steigender Diphthonge aus den fällenden in ihrer reduzierten Form nachweisbar ist und dass im konkreten Falle einer solchen Ableitung keine formalen, sonderm nur semantische Schwierigkeiten im Wege stehen, da vom etymologischen Standpunkt her nicht klar ist, an welche ei- bzw. ai- Wurzel das Wurzelmorphem jia- anzuknulpfen wäre. Nun liegt es nahe, fur alle hier beschriebenen Manifestationen einer I- oder I-ähnlichen Charakteristik den Grund in irgendeiner, obgleich nichtauditiven Nachahmung des realen Phänomens "hell" zu suchen. Im Einklang damit wird das italienische synonym: brillante tatsächlich von der herrschenden Meinung auf ein romanisches, als imitativ bezeichnetes Morphem birl- zurickgefinrt. Indessen ist die Frage uber die Art der Nachahmung nicht leicht zu beantworten, und die Forscher, die It. brillante als imitativ erklärten, sind offenbar mehr ihrem instinktiven sprachgefunl als einer umfassenden kritischen Analyse des vorausgesetzten Zeichenprozesses gefolgt. Aus der traditionellen etymologischen Untersuchung kann man hier wie in anderen ähnlichen Fällen keine nähere Information uber den ikonischen Zeichenprozess erhalten, 
so dass die Aufstellung eines imitativen birl- nicht objektiv zwingend erscheint. Durch die statistische, quantitative lifethode bei der zusammenfassenden Beobachtung von ganzen Synonymen serien sind wir jedoch im Stande, uber die Kontroverse hinaus, ob wir es mit einem neugeschaffenen lexikalischen Element oder nur mit einer Umdeutung des ubernommenen griechischen Fremdwortes: beryllos zu tun haben, festzustellen, dass es sich in beiden Fällen um ein ikonisches sprachzeichen handelt, sei es primärer oder sekundärer Natur. Das geht mit grösster Wahrscheinlichkeit aus dem immer von neuem wiederholten parallelismus der I-Diphthonge in der Serie und der zusätzlichen Tatsache hervor, dass im Vulgärlateinischen wegen des regulären Lautwandels kein Synonym mit der I-Charakteristik iberlebte; das angefuhrte lat. nitidus wandelte sein $I$ in $E$ um und ergab It. netto, Fr. net. Nur die Frage nach der inneren Struktur des zugrunde liegenden Zeichenprozesses bleibt, wenn wir uns ausschliesslich auf die Analyse der isolierten serie fur "hell" beschränken, weiterhin schwer beantwortbar. Man könnte vermuten, dass die überzäligen I-Charakteristiken durch die bekannte, auf der Synästhesie beruhende Relation zwischen "hell" als optischem Phänomen und I als "hellem" Vokal begründet sei. Aber in diesem Falle müsste man die Synästhesie nicht nur als bestimmenden Faktor bei der semantischen Umdeutung vorhandener lexikalischer I-Formen, wie eventuell bei: brillante, bzw. spezieller phonematischer Umformung lexikalischer Formen mit andersartigem Vokalismus zu I-Formen, wie das bei Ae. briht aus berht stattgefunden zu haben scheint, also bei der aktiven Verarbeitung lexikalischen Materials zu sekundären Ikonen annehmen. Man müsste die synästhestische Einwirkung als treibende Kraft sogar bei der Konstituierung und primären Erweiterung der als urspringlich geltenden indoeuropäischen Wurzelelemente betrachten, was eine wortschöpfende Macht dieser an sich noch unklaren psychophysiologischen Erscheinung verlangen würde. Nach unserer bisherigen Kenntnis kommt die Synästhesie nur als nachträglicher, sprachinterpretierender, und nicht als sprachverändernder Faktor ins 
Spiel. Sie kann bej irgendwelchen lexikalischen I-Formen auftreten, wie bei den genannten so auch bei den automatisch durch den regulären Irautwandel enstandenen; so z.B. bei Nhd. Ijcht, bei dem wir genau wissen, dass es auf ein Wurzelmorphem leukzurikckgeht mit der konstanten Bedeutung "hell" im historischen Zeitverlauf, die keine Vermutung uber eine aktive synästhetische Beeinflussung zulässt.

Den richtigen Fingerzeig fur eine positive Antwort gibt uns die parallele Analyse einer der Serie "hell" sehr ähnlich strukturierten Synonymenreihe, d.i. der Serie IOOK, IOOK AT /B 15.52/. Die Serie enthält, wenn wir 4 Wurzelmorpheme mit dem U-Anlaut dazurechnen, 22 etymologisch erschlossene Wurzelmorpheme nebst 8 etymologisch unerklärten Synonymen. Von den 18 Morphemen der Grundzählung besitzen 8 eine I-Charakteristik: k'ei- uber Gr. koíte zu Ngr. koitadzō, /s/mei- uber Lat. mírārī.zu It. mirare, Ls/lei- uber tat. oblivisci, oblitus zu Rum. se uita, $\mathrm{k}^{\mathrm{w}}$ ejzu Ir. ad-cīu, dēccu, stilp-, stilb- zu Ir. sellaim, gei- zu Nl. kijken, dei- zu Tschech. divati se und dheig- zu Av. di-. Wie wir sehen, haben 7 von den aufgezthlten Morphemen eine diphthongische und eine von ihnen eine reine I-Charakteristik, zusammen betragen sie aber viermal mehr als die Durchschnittzahl ergeben würde. Von den 4 Morphemen mit dem U-Amlaut weisen weitere 2 die I-Charakteristik auf: weid- zu Iit. veizdèti im eigentlichen Wurzelkem und wel-, wlei- zu Got. wlaitōn, Ae. wiztan in der Wurzelerweiterung. Da unter den Wurzelmorphemen mit steigendem U-Diphthong in der Morphemsammiung unseres Musterbeispiels die I-Charakteristik kaum bei jedem zweiten Morphem sich finden lässt, ist auch hier eine von ihnen uberzählig. Dazu tritt noch das $I$ in der Erweiterung des Grundmorphems bhel- zu Nhd. blicken und in 2 der ungeklärten synonyme: Dăn. titte und Ne. peep. Das Verhältnis der I-Formen zu den anderen ist ungefähr dasselbe wie in der Serie für "hell", die ungewöhnliche Anhäufung von ihnen jedoch auf den ersten Blick noch rätselhafter wie dort. Besonders $z u$ beachten ist, dass neben den 
I-Diphthongen ein Morphem stilp-, mit einem primären I auftritt, das dem Bau indoeuropäischer Wurzeln, die vor einem wurzelhaften I durchgehends kein I dulden, ausdrticklich widerspricht. Auch haben wir in dem skandinavischen: titte, titta und dem englischen: peep allem Anschein nach den gleichen Fall von sekundärem Ikonismus, wie er uns bei dem italienischen: brillante begegnete. Endlich ist in der zeitlichen Entwicklung das I bei den Synonymen fur "ansehen" im allgemeinen sogar besser erhalten bzw. wird ofter erreuert als bei den synonymen für "hell". Doch, wenn eine Erklärung durch Synästhesie schon dort höchst fraglich erschien, so ist diese, weil sie eine Verbindung zweier Qualitäten darstellt, im vorliegenden Falle, wo es um ein ausgesprochenes Tätigkeitsphänomen geht, völlig ausgeschlossen. Der Schlussel zur Lösung des Problems liegt in der semantischen Analyse beider Serien. Es ist den Etymologen schon lange bekannt, dass die Bedeutungen "sehen", "ansehen" und" "glänzen" nicht selten unter den gleichen Wurzelmorphemen zu finden sind; beiden Synonymenreihen sind 4 solche Morpheme gemeinsam, dei- in Ai. dyumantund Tschech. divati se, bhel- in Dän., Schw. blank und bhleiin Nhd. blicken, g'hel- in NIr. geal, Nhd. glänzend, sowie g'hlei- in NIr. glēineach und wiederum g'hel- in Kslav. ględati, dann aber auch leuk- in Iat. lücidus und in Gr. lebisso, Lett. lūkuot. Die Liste gleichartiger polysemantischer Morpheme liesse sich bemerklich erweitern, da die Serien in Bucks wörterbuch nicht als erschöpfend angesehen werden können und auch nicht alle indoeuropäischen sprachen darin vertreten sind und noch weniger ihr dialektischer Wortschatz. Einstweilen greife ich nur 2 weitere aus der Masse heraus, welche in je einer serie der beiden hier behandelten erscheinen, aber in der anderen fehlen. So hat An. skāērr "hell" sein Gegenstück in An. skim- "spähen, sich umsehen" vom gemeinsamen Wurzelmorphem sk' $\bar{a} i$; und Ir. sellaim "ich sehe an", das zum wurzelmorphem stilp-, stilb- gehört, hat umgekehrt seine semantische Ergänzung im verwandten Gr. stilp-nos "glänzend". Es ist noch hinzuzufügen, dass neben Dän., Schw. blank auch ein bedeutungsnahes Ae. blïthe- "heiter, 
freundlich" /Ne blithe/ mit der I-Charakteristik existiert, und ein I-charakterisiertes Norw. dial. glina "glänzen, stieren" neben Kslav. ględati. In den Paaren dyumant - divati se, skāērr - skima, stilpnos - sellaim, blithe - blicken, glēineach - glina zeigt sich nun ein vollständiger Parallelismus zwischen der Doppelbedeutung "hell" - "ansehen" einerseits und der I-Charakteristik andererseits, welcher die ungewöhliche Anhäufung von IMorphemen in Bucks Serien für "hell" und fur "ansehen" erklärt, obgleich bei vielen von ihnen die gleiche semantische struktur nicht nachweisbar ist. Die Rechtfertigung dieser Behauptung liegt im direkten Vergleich der Sprachzeichen mit den bezeichneten Phänomenen. Bei der Einwirkung der Helle auf unsere Augen, was man besonders in Fälien des jähen übergangs von Dunkeln zum Hellen oder gesteigerter Lichtintensität beobachten kann, näherm sich die Augenlider und ziehen sich in die länge, so dass sie die gleiche Form annahmen wie die lippen bei der Artikulation des I-Lautes. Die durch den Iichtreiz entstandene Bewegung der Augenlider und das Resultat der Bewegung werden, wie uns die Physiologie lehrt propriozeptiv empfunden, ebenso wie bei der Artikulation des I-Lautes propriozeptive Empfindungen auftreten; bei der objektiven Ähnlichkeit beider Bewegungsverläufe können wir mit ziemlicher Sicherheit vermuten, dass der objektiven Ähnlichkeit auch die subjektive Wahrnehmung dieser Ähnlichkeit entspricht. Ausser der propriozeptiven Wahrnehmung am eigenen Körper besteht noch die optische Wahrnehmung der Vorgänge in den Augen und am Mund bei anderen Personen und bei der Selbstbespiegelung, so dass die Annahme einer artikulatorisch-mimischen IIkone für "ansehen" und gleichzeitig für "scheinen" und "hell". als methodisch vollständig befriedigende lösung des Problems der überzähligen I-Charakteristiken in unserem Falle erscheint. Es muss sogar auf die Möglichkeit der Auffassung der I-Ikonen als potentielle I-Indexe im sinne von C.S.Peirce hingewiesen werden; es bleibt nämlich durch nervenphysiologische Untersuchungen festzustellen, ob nicht bei besonders starker Verengerung der Augenlider oder bei besonders starker Artikulation 
eines I nicht Synergie zwischen der Augenmuskulatur und der Mundmuskulatur besteht. Bei einer solchen Feststellung müsste man die I-Ikonen geradezu als physiologisch provoziert betrachten.

Die Annahme eines artikulatorisch-mimischen Zusammenhanges bei den I-Morphemen für "ansehen", "scheinen", "hell" kann jedoch auch ohne die interdisziplinäre Ausdehnung der Untersuchung durch die weiterentwicklung der morphosemantischen und realitätsvergleichenden Analyse der Synonymenreihen bekräftigt werden. Zuerst holen wir die quantitativen Daten fur SHINE unter Bucks fortlaufender Nummer 15.56 nach: 9 Wurzelformen mit I, teils in den Grundformen, teils in den Wurzelderivativen - ein solches mit UI-Anlaut inbegriffen - stehen 9 andersartige Wurzelformen, davon zwei U-Morpheme, die wir schon bei der Serie "hell" angetroffen hatten, entgegen. Von den 4 nicht zu zählenden restlichen Synonymen ist eins das strittige italienische: brillare, 3 sind unerklärt, unter denen sich wieder eine I-Form vorfindet: Lit. žibeti. Wenn wir uns nun die immer wiederkehrenden I-Norpheme systematisch auf ihren ganzen Sinngehalt hin ansehen, entdecken wir bei mehreren bisher unerwähnte mimische Bedeutungen. Unter nei-, das in Lat. nitidus, nitēre auftritt, finden wir, den Angaben aus etymologischen und lexikalischen Standardwerken folgend, ein gleichsprachiges: renidēre; dieses ist in der Bedeutung "glänzen" mit nitêre identisch, steht aber auch für "lächeln", "vor Freude strahlen" sowie für "höhnisch lächeln", "grinsen", das den vorhergehenden Bedeutungen auf den ersten Blick geradezu entgegengesetzt erscheint. Das gleiche Bedeutungsverhältnis finden wir unter dem wurzelmorphem $\angle \mathrm{s} / \mathrm{mei-}$, das über Lat. mīrāri in It. mirare "ansehen" auftaucht; in den meisten indoeuropäischen und abgeleiteten Sprachen liefert es die Bedeutung "lächeln", wie z.B. in Ne. smile, im Lett. jedoch wiederum daneben auch "verlachen", "spotten", wie sie in den Verben: smiêt, smaidit erscheint. Dasselbe begegnet uns bei den Ablegern der erweiterten Wurzelform g'hlei-. Das schon angefühte Neunorw. glina "glänzen", "stieren" hat neben sich ein Schw. glina 
"lächeln" und eng verwandte Formen mit -s-: Neunorw. glĩsa

"schimmern, durch eine schmale Öffnung leuchten, die Zähne zeigen, hohnlachen", Schw. dial. glisa "schimmern, gucken, foppen, hohnlachen", denen sich noch Formen mit $-r$ - anreihen: Neunorw. glira "mit den Augen blinzeln, gucken, schimmern, leuchten, höhnisch lachen" sowie Schw. dial. glira "blinzeln". Das Nebeneinander aller dieser so verschiedenen Bedeutungen, das für die traditionelle Semasiologie ein schwer entwirrbares Problem darstellt, erklärt sich mühelos, wenn wir bedenken, das beim Iächeln wie beim höhnischen Grinsen die Jippen und der ganze Mund in der gleichen Weise auseinandergezogen werden und dass dieser mimische Ausdruck genau einer intensiven Artikulation des I-Lautes entspricht. Dabei vergessen wir nicht, dass wir bei unserer Analyse von den Serien "ansehen", "scheinen", "hell" ausgegangen sind und dass es daher nicht verwunderlicht ist, wenn wir erneut auf diese Bedeutungen in den neu angeführten Wörtern stossen. Die I-Ikonen für "lächeln" und "grinsen" bestätigen die Existenz der I-Ikonen für "ansehen" usw., wie umgekehrt. Dass nun die gleiche Charakteristik zur Bezeichnung realer Phänomene gebraucht wird, die zwar ein gleiches bzw. sehr ähnliches reales Grundmerkmal besitzen, aber doch verschiedene Phänomene darstellen, ist aus der kleinen Anzahl der klar unterscheidbaren Phoneme verständlich, die für die ikonische Bezeichnung in der Sprache zur Verfugung steher. Nachdem wir zur Feststellung des Polyikonismus einiger typischer polysemantischer I-Morpheme über die Grenzen von Bucks wörterbuch hinausgegriffen haben, werfen wir noch einen kurzen Blick auf die von ihm registrierte Serie SMILE /B 16.25b/. Von 15 untereinander unabhängigen Synonymen gehören 11 bekannten Wurzelmorphemen an, wobei 2 Morpheme mit U-Anlaut eingerechnet sind. In diesen 11 Wurzelformen tritt 4 mal eine I-Charakteristik auf, $3 \mathrm{mal}$ in der Grundform und ImaI in der Wurzelerweiterung: in /s/mei- u Gr. meidiāo, Ne. smile, Lett. smaidit usw., in mei- zu Nir. mionghāirim, in k'eip- zu Iit. šypsotis und in wer-, wrei- zu Lat. subrīdēre und seinen romanischen Vertretern. Das $I$ ist offenbar überzählig, obgleich die vorher zitierten Synonyme: Lat. renidēre und Schw. glina in 
Bucks Liste nicht vorkommen. Alle hier neu erscheinenden Wurzelmorpheme sind polysemisch oder haben homophone Wurzelmorpheme als Begleiter in dieser Polysemie bzw. Homophonie sind weitere I-Ikonen $z u$ entdecken, was uns jedoch $z u$ weit führen wirde. Es genügt, auf eine interessante sekundäre I-Ikone hinzuweisen, die in der Serie auftritt. Das holländische Synonym für "lächeln" ist: glimlachen, eine Substitution für grimlachen, bei Buck durch den Einfluss von: glimmen "twinkle, glimmer" erklärt. In Wirklichkeit handelt es sich um die metaphorische übertragung einer mimischen Ikone auf die andere; das "strahlende" Lächeln wird eigens durch den I-Vokalismus charakterisiert.

Ich habe mich vorsätzlich bei der Beschreibung und Erläuterung des Ikonismus in den untersuchten Synonymenreihen länger aufgehalten als ihnen das im festgesetzen Rahmen in Hinsicht auf andere noch zu behandelnde zusteht. Doch mögen sie als Modell aller unserer Analysen dienen und als geeignetster Prifstein ihrer Gültigkejt. Weiterhin werden sich die speziellen Analysen der einzelnen Serien notgedrungen nur auf das Wichtigste beschränken müssen. Betrachten wir als Gegensatz zum Vorhergegangenen eine Gmappe von Serien mit überdurchschnittlicher U-Charakteristik. Die Serie KISS /B 16.29; Iing. IX/I,23/ besteht aus 19 Synonymen; 2 davon sind unbekannten Ursprungs und 3 gelten als einzelsprachliche Onomatopöien. Von den 14 synonymen mit bekannten primären Wurzelmorphemen besitzen 5 eine U-Charakteristik und I entstammt einem Morphem mit steigendem U-Diphthong. Das sind Gr. kunēo, ON kyssa usw. zu kus-, Lat. ōsculäri zu ōus-, Rum pupa uiber ein rekonstruiertes vlat. * puppa zu pü-, Lit. bučioti, Nhd. Buss usw. zu bu-, Skr. ljubiti zu leubh-; daneben Lat. sāvī̄ri zu syād-. Wenn man steigende Diphthonge in die Zählung einbezieht, würde der Durchschnitt 3, sonst 2 U-Morpheme betragen. Beachtenswert darüber hinaus ist vor allem zweierlei: erstens haben alle drei als einzelsprachliche Onomatopöien geltende synonyme, d.i. Got. kukjan, Lett. skūpstīt und Ai. cumb- die gleiche U-Charakteristik, und zweitens werden mit Ausnahme der Morpheme ous- und leubhauch alle primären Wurzelmorpheme mit $U$ in der etymologischen 
Iiteratur als onomatopoetisch angesehen. Unsere quantitative Analyse und die traditionelle stellungmahme stimmen also im Prinzip, dass die Lautstruktur der Serie durch Sprachikonen bedingt sei, verblüffend uberein. In einer weiteren Serie, BIOW "blasen"/B 10.38; Ling. VIII/2, 143/ uberwiegt die U-Charakteristik sogar absolut. Von 8 unabhängigen Synonymen, die alle eine anerkannte Etymologie haben, entstammen 5 U-haltigen wurzelmorphemen, 1 geht auf einen steigenden U-Diphthong zurück. So haben wir pneu- zu Gr. pnēo, bh/e/u- zu Gr. phūsłō, au/e/zu Gr. "äemi, pü- zu Iit. püsti und dheu- zu Kslav. duchati; ausserdem findet sich suei- vor zu Ir. sētim. Bei einer engeren Zählung dürfte im Durchschnitt nur ein U-Morphem auftreten, mit Einbeziehung der steigenden Diphthonge höchstens 2. Auch hier handelt es sich mit Ausnahme von au/e/- und dheu- um anerikannte onomatopoetische Morpheme, so dass die Signifikanz der ungewöhnlichen Anhäufung der U-Charakteristiken in der Serie von der aussenstatistischen Seite her bestätigt wird. Wie schon anfangs bei der Analyse der Synonymenreihe für "sprechen" treffen wir nun auf eine identische charakteristik bei unbezweifelten Onomatopöien und den als konventionell betrachteten Sprachzeichen, und wiederum drängt sich die Frage auf: ist das ein Zufall? Stellen wir die Antwort noch einmal zurück. Der Vorrang gebührt nämlich einer anderen Frage: geht es bei den als onomatopoetisch betrachteten Sprachikonen wirklich um Schallnachahmung, um Reproduktion auditiver Wahrnehmungen? Die Erklärung im Standardwerk von J. Pokorny beim Wurzelmorphem b/e/u-, bh/e/u- /p 98/: Sprenglaut der aufgeblasenen Backe, und beim wurzelmorphem püpeu- /P 847/: Schallvorstellung der aufgeblasenen Backen, zeigt, dass die Entstehung dieser Morpheme von den Etymologen als Nachahmung einer hörbaren menschlichen oralen Geste aufgefasst wird. Parallel dazu wird das Wurzelmorphem bu- aus der Serie für "kiissen" als die Nachahmung der Sprengung des saugenden Lippenverschlusses nach innen, also einer anderen oralen Geste, festgestellt. Wir haben keinen Grund, an der richtigen Auffindung der durch die Sprachikonen ursprünglich bezeichneten Realität 
zu zweifeln. Jedoch, die Auffassung des Zeichenprozesses und die Kategorisierung der angefuhrten Morpheme sind nur teilweise begriundet und daher falsch; völlig unzureichend ist die blosse Erwähnung einer Schallnachahmung bei den synonymen wurzelmorphemen pneu- bzw. kus-. Von Schallnachahmung kann nämlich bei allen Ikonen für "blasen" und "kỉssen" nur im Hinblick auf die Konsonantencharakteristiken die Rede sein, besonders auf die Labialcharakteristik; es ist unötig, experimentell nachzuweisen, dass das $U$, das dea Ikonen gemeinsam ist, mit Schallnachahmung nichts zu tun hat. Gerade auf dieses immer wieder sich wiederholende U sind wir aber durch unsere quantitative Analyse ausdrucklich hingewiesen worden. Man braucht sich nun nur die aufgeblasenen Backen und die dazugehörige Mundform einerseits und den saugenden Lippenverschluss andererseits lebhaft vorzustellen, um in diesen oralen Gesten die grösste Ähnlichkeit mit der Artikulation eines gerundeten $U$ zu erkennen. Wir können schliessen: die behandelten Serien zeugen von einem Mischtypus teils onomatopoetischer, teils artikulatorischer Ikonen, in dem die U-charakteristik bestimmt die artikulatorische Komponente darstellt, während der Nachahmungsprozess, die Konsonantencharakteristiken betreffend, noch zu untersuchen ist. Der nächste schritt fuhrt uns zu einer dritten Serie mit uberzähliger U-charakteristik; es darf uns nicht verwunderm, wenn dies die Serie MOUTH ist /B 4.24: Ling. IX/1,22/. Alle 14 Synonyme gehören bekannten Wurzelmorphemen an; 5 davon sind U-Morpheme und 2 enthalten einen steigenden U-Diphthong. Wir wollen sie aufzählen: ous- zu Lat. ōs, b/e/u-, bh/e/u- ubber lat. bucca zu It. bocca, mûu- zu Lett. mute, Ai. mukha-, gēu- zu Pol. geba und au- zu Ai. vadana-. Mit einem steigenden U-Diphthong hingegen: guet- zu Ir. bēoil und uek ${ }^{\mathrm{W}}$ - zu Ai. vaktra-. Das Verhältnis derDurchschnittszahlen zu den auftretenden ist bei einer engeren Zählung 2 zu 5, mit Einbeziehung der steigenden Diphthonge 3 zu 7. Dazu kommt noch das russische synonym: rot, dessen Wurzelform nach Buck eine I-Erweiterung von er- sein soll, wahrscheilicher jedoch nach Vasmer, Miklošič u.a. einer Form ereu-, reu- mit $U$ in der Wurzelerweiterung bzw. im Wurzelvokal entstammt. 
Obgleich ausser b/e/u- in dieser Serie nur das Wurzelmorphem $\underline{\underline{u} \text { - }}$ als lautilich motiviert anerkannt wird, haben wir offenbar hier mit einer ikonisch weitgehend bedingten serie zu tun, und zwar diesmal, da es um ein unhörbares reales Phänomen geht, mit einer feihe rein artikulatorischer Ikonen. Es lohnt sich, daran zu erinnern, dass W. Wundt, ein Zeitgenosse de Saussures, der schon am Anfang dieses Jehrhunderts die Kategorie der Iautgebärden als unwillkürlicher Nachahmungen der Bewegungen durch den Laut aufstellte, insbesondere auf die Existenz solcher Lautgebärden hinwies, die sich auf die sprachorgane und ihre Tätigkeiten beziehen. Unsere empirische statistische Untersuchung bestätigt vollauf diese seine Erkenntnis. Wir haben in allen drei Serien für "kiissen", "blasen" und "Mund" artikulatorische Organikonen bzw. Organfuntionsikonen vor uns, leicht vermischt mit onomatopoetischen Elementen, die jedoch in der serie für Mund gänzlich zürucktreten. Sehen wir uns die Serie noch einmal vom synchronischen. Standpunkt an. Das Morphem ous- hat in Lat. ōs sein U verloren, doch es bleibt im dazugehörigen Skr., Tschech., Pol. usta erhalten. Das $U$ von $b / e / u$ - ist in It. bocca in 0 umgewandelt, doch es erscheint wieder in Fr. bouche. Einem unveränderten primären $U$ begegnen wir in liett. mute, während das $U$ von gēu-, welches in Pol. gęba keine Spur hinterlassen hat, in den tschechischen und serbokroatischen Schimpfnamen für Mund: huba, gubica zu Tage tritt. Von den 5 U-Charakteristiken in den Wurzelmorphemen leben also 4 in ihren modernem Ablegern weiter; dabei ist das $U$ in Frz. bouche durch regelmässigen Lautwandel erneuert. Daneben treten aber in den modernen Sprachen noch drei Synonyme mit der U-Charakteristik auf: Rum. gură, das über Iat.

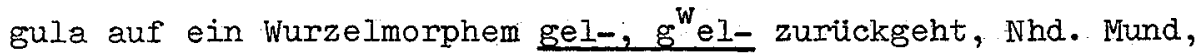
eine schwundstufige Ablautform des Wurzelmorphems menth- und Lit. burna von einer reduzierten Form der Wurzel bher-. Eine offenbare verschiebung hat indessen nur bei Rum. gură aus "Schlund" zu "Mund" statgefunden, ähnlich jener bei It. bocca, Fr. bouche aus "/aufgeblasene/ Backe". Wie sollen wir das Auftreten des U, das nicht in den primären Wurzelmorphemen gegeben war, beurteilen? 
Rum. gură ist allem Anschein nach eine sekundäre U-Ikone, eigentlich eine metaphorische Übertragung einer solchen von der Bezeichnung des Schlundes zur Bezeichnung des Mundes gerade der semantische Prozess der metaphorischen bzw. metonymischen Übertragung zeugt davon. Heute ist zugleich mit dem Bewusstsein davon auch das Gefühl der ikonischen Nachahmung gewiss tot, gleicherweise abwesend wie in Nhd. Mund und Lit. burma, was jedoch an der einstigen Ròlle des Synonyms als einer sekundären Ikone richts ändert. Beim deutschen und litauischen synonym bleibt uns indessen zum Kriterium nur die Form. Und da stellt sich die Frage: war die Wahl der schwundstufigen Wurzelform für das gemeingermanische: Mund zufällig? Warum wurde für die litauische Bezeichnung des Mundes eine 0-haltige Reduktionsform gewählt, die $U$ als Ergebnis hatte?

Nur im Rahmen unserer Serie, ohne zusätzliche semantische Daten und ohne die Gelegenheit eines interseriellen Vergleichs ist eine annathernd befriedigende Antwort dieser an sich berechtigten Fragen unmöglich. Bisher haben wir solche breitere Untersuchungen, die über die grundsätzliche Feststellung artikulatorischer Ikonen hinausgehen, absichtlich unterlassen. Wenn ich jetzt den weg zur Antwort auf die Frage hinsichtlich des deutschen synonyms: Mund zu skizzieren versuche, so geschieht das vor allem, weil wir hier wirklich auf neue Arten des artikulatorischen Ikonismus stossen und dabei an einem nahen Beispiel die angewandte quantitativ-qualitative Methode zur lösung semiotischer Probleme näher dargelegt werden kann. In der veröffentlichten Bearbeitung der Serie ist das Wort: Mund im Einklang mit der Mehrheitsmeinung der Etymologen auf das Wurzelmorphem menth- zuruickgefüht, andere ziehen ein verschiedenes, als unverwandt betrachtetes menvor. Jedoch in beiden Fällen hat: Mund eine Bezeichnung für "Kinnlade", "Kinnbacke" neben sich: sei es Gr. mbthuiai bei menth- oder sei es ein älteres Cymr. mant bei men-. Wir sehen uns die Serie JAW /B 4.207; Iing. VIII/1,9/ an und finden zwar keines der zitierten, bei J.Pokorny bezeugten wörter, hingegen wohl das Wurzelmorphem menth-, das dem frz. Synonym machoire über 
Lat. masticäre von Gr. mastichăō zugeordnet ist. Zu Gr. génus; gnathos finden wir ein weiteres Wurzelmorphem mit einer N-Charakteristik: E'enu-, das wiederum auch in der serie für "Mund" bei den keltischen wörtern: Ir. gin usw, auftritt. Noch eine weitere Verbindung beider Serien durch die N-Charakteristik stellt das Wurzelmorphem stomen- vor, in dem das Gr. stoma und das Cymr. safn "Kinnlade" vereint werden. Nur in der serie für "Kinnbacke" erscheint indessen ein viertes gleicherweise N-charakterisiertes Morphem smek-, das dem Lat. māla, maxilla, It. mascella usw. entstammt. Nun ist diese $\mathrm{N}$-Charakteristik eine ikonische artikulatorische Bezeichnung fur die zusammendrickende Bewegung der Kinnlade bzw. des Mundes, wobei neben den äusseren beim Kauen beteiligten Muskeln auch die Muskeln im Innerm des Mundes durch den spannenden. Verschluss des Velums und der Lippen mitwirken. Der statistische Test der Signifikanz der Nasalcharakteristik im morphosemantischen Bedeutungsfeld des Drückens ist mittels einer 3 gliedrigen Gruppe von Synonymenreihen durchgeführt worden; von den einzelnen serien mit besonders auffälligem Auftreten der N-Charakteristik möchte ich auf die Serie KNEAD /B 5.54; Iing. VIII/1,10/ hinweisen, wo die artikulatorisch-manuellen Ikonen des Drückens doppelt den normalerweise zu erwartenden N-Durchschnitt ubersteigen. Auch diese Ikonen stellen potentielle Indexe dar, da grosse Wahrscheinlichkeit einer synergischen Mitwirkung der Muskeln in der Mundregion und den Handmuskeln besteht. Da wir nun den Ursprung des Wortes: Mund in einer primären $\mathrm{N}$-Ikone des Zusammendrïckens der Kieferm und Lippen entdeckt haben, haben wir aber auch gleichzeitig feststellen können, dass das Auftauchen des U-Vokalismus in unserem Synonym nicht in enier ikonenbildenden Tendenz zu einer neuen sekundären. U-Ikone zu suchen ist. Denn sowohl Cymr. mant, welches neben "Kinnlade" selber "Mund" bedeutet, wie auch Gr. máthiuai, das von einem nahverwandten: mástax "Mund" begleitet wird, gehen auf eine gleiche schwundstufige Form mn- bzw. mpthzuriuck wie das germanische-Mund. Wie dort das $U$, ist hier das A streng lautgesetzlich und die Tendenz zur Erreichung einer 
bestimmten Form, wie sie bei jeder Ikone vorauszusetzen ist, konnte nicht einseitig nur bei einem worte oder gleichzeitig in zwei verschiedenen Richtungen arbeiten. Wir haben nämlich, trotzdem das aus Bucks Serie nicht zu ersehen ist, mit einem zweiten vokalischen Ikonismus bei den Bezeichnungen des Mundes zu rechnen, d.i. mit dem Ikonismus des weit sich öffnenden und dann zusammenschlagenden Mundes. Auch diesartige Ikonen sind der traditionellen etymologischen Wissenschaft längst bekannt, wobei sie freilich vornehmlich als Onomatopöien gedeutet werden. Das sind vor allem die Wurzelmorpheme kap- und ghabh-; das subjektive Bewusstsein der. Ikone ist im Deutschen durch die interjektion happ! zum Verbum happen, hapsen bezeugt; die germanische intensive Konsonantengemination und das Aufnehmen der schriftdeutschen Interjektion aus dem Niederdeutschen sind gewiss nicht zufällig. Wenn Buck in seinem Synonymenwörterbuch bei der Serie für "Mund" keine Synonyme mit dem A-Vokalismus anfihrt, ist das seiner prinzipiellen Stellungnahme zuzuschreiben, alle Vulgämamen fir den Mund, insoweit sie nicht Normalwörter geworden sind, auszulasen. Dabei ist notwendigerweise der Ausblick auf die Phonemstruktur der Synonyme unvollständig geblieben. Ohne mich mit den Etymologien und näheren statistischen Erwägungen $z u$ befassen, zitiere ich als Beispiel aus der Liste der. Mundsynonyme, die Dornseiff in seinem Wörterbuch deutscher Synonyme zusammengestellt hat. Auf der einen Seite hat Dornseiff: Mund, Brutsche, Futterluke, Maul, Muffel, Muppe, Nuge, Nusche, Schnull, Schnauze, Schneuel, Schnure, Schnute mit dem U-Vokalismus, auf der andere Seite aber: Haffel, Raffel, Schnabel, Klappe, Flabbe, Labbe mit dem A-Vokalismus, während Formen mit I-Vokalismus nicht auftreten. Da es sich bei den vulgären Synonymen für "Mund" meist um Schimpfwörter handelt, die nebenbei einen Vergleich mit dem tierischen Mund enthalten, möchte ich darauf aufmerksam machen, dass dieser Mund sich nach seiner anatomischen Bauart und wegen Abwesenheit der menschlichen Mimik wirklich nur zur ikonenhaften Darstellung mittels eines runden, vorgestiulpten $U$ oder eines intensiv, mit 
weitaufgerissenen Kiefern artikulierten A eignet. Aus dieser Perspektive können wir noch einmal auf den Ausgangspunkt zurückblicken, der uns zur erweiterten Analyse der Mundsynonyme gefuhrt hat. Die dort gestelite Frage muss umformuliert werden. Wir missen fragen, nicht, ob die wahl des germanischen Synonyms: Wund, des cymrischen: mant und des griechischen: mástax absichtlich in einer schwundstufigen Form erfolgte, sonderm, warum uns die Bedeutung "Wund", ausgedrickt durch die Morpheme mnthbzw. m-, nur im germanischen, keltischen und griechischen Sprachbereich iberliefert ist, wo U- und $\Lambda$-Vokalismus als Entwicklungsresultat lautgesetzlich auftreten, nicht aber z.B. im Litauischen und Lateinischen, wo die gleiche Lautgesetzlichkeit zu Formen mi -iñ-bzw. -en- führen muisste. Cymr. mant hat im Lateinischen zwar ein formell genau entsprechendes Gegenstück: mentum, es bedeutet jedoch "Kinn" und nicht "Mund". Warum, hingegen, hat das Lateinische im engverwandten Worte fiur "kauen" ein $A$ in: mendere statt * mendere, wie die lautgesetzliche Entsprechung zum gleichbedeutenden Gr. masáomai lauten müsste? Auch Lat. mäla, maxilla enspricht keiner normalen Ablautstufe des Wurzelmorphems smek-. Warum, können wir weiterfragen; hat das cymrische: sain zum Wurzelmorphem stomen- ein abnormales $A$ und nicht das lautgesetzliche O? Und endlich: das Gr. gnáthos welches sich vom gleichsprachigen: genus auch dadurch unterscheidet, dass es nur "Kinnbacke" und nicht auch "Kinn" bedeutet entsprichi zwar der lautgesetzlichen Entwicklung einer indoeuropäischen Schwundstufe, doch woher von neuem die Schwundstufenform im unerklärten Derivativ? Dürfen wir nicht eine ikonenbildende, und wo nicht diese, eine ikonenerhaltende Tendenz bei allen diesen lautlichen und semantischen Erscheinungen als mitwirkende Kraft annehmen? Die Sprachen der indoeuropäischen Familie haben wegen ihrer frühen und reichen schriftichen Tradition und der mihevolien Arbeit mehrerer Generationen von Linguisten für das Studium des Ikonismus den grossen Vorteil, dass in ihnen die ikonischen Zeichenprozesse auch in der Diachronie beobachtet werden können; damit wird die Möglichkeit 
gegeben, die Erscheinung des Ikonismus in seiner engen Verwobenheit mit der ganzen Sprachgeschichte zu erkennen.

Mit den artikulatorisch-mimischen Ikonen und den artikulatorischen Organ- bzw. Organfunktionsikonen haben wir zwei grundlegende Kategorien der nichtonomatopoetischen Sprachnachahmung beschrieben, die direkt an die Sprachorgane gebunden sind. Die Charakteristiken dieser Ikonen brauchen freilich nicht nur vokalisch zu sein; ich habe mich hier mit dem Vokalikonismus befasst, da gerade dieser wichtige Teil meiner Studien hier zum ersten Mal erläutert wird, während die Bearbeitung des Konsonantismus, nicht vollständig zwar und stellenweise provisorisch, im Druck zugänglich ist. Die weiteren Ikonenkategorien sind in der Regel Übertragungen, auf einem unbewussten Mechanismus der Form- und Tätigkeitsvergleiche fussend, was gewiss ein Hindernis sogar fïr ihre wissenschaftliche Erkennung darstellt. Eine davon, die Kategorie der artikulatorisch-manuellen Ikonen, haben wir nebenbei an dem Beispiel KNEAD "kneten" erwähnt. So ist auch die aus primären Wurzelmorphemen ersichtliche A-Charakteristik in Bucks wörterbuch am besten bei Serien mit artikulatorischmanuellem Ikonismus bezeugt. Die Serie SEIZE, GRASP, TAKE HOID OF /B II.14; Iing. VI, 3I/ enthält von 23 unabhängigen primären indoeuropäischen Wurzelmorphemen 6 , die eine ursprïngliche ACharakteristik aufweisen: labh- zu Gr. lambsnō, kap- zu Iat. capere, sāg- über ein Fränk. *sakjan zu Fr. saisir, ghabh- zu

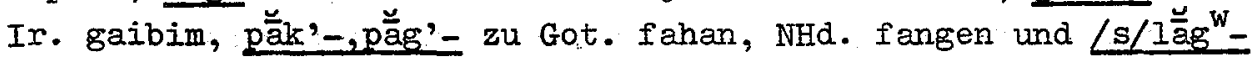
zu Ags. laeccan. Das beträgt das Doppelte der Durchschnittzahl. Von den. 4 ungezählten Synonymen beruht das tschechische: uchopiti auf einem slavischen imitativen chop-, das mit seinen Varianten in Russ. chabit', Ukr. gabaty usw. auch auf ein ursprungliches A hinweist. Das unerklärte Span. angarrar andererseits wird auf eine iberische oder gallische form *garr- zurïckgefuhrt. Der ikonenhafte Charakter der anerkannten imitativen Morpheme tritt ausser dem A besonders im labialen Auslaut hervor; zu beachten ist der gleiche labiale bzw. der ähnliche labialisierte Auslaut in den parallelen Wurzelmorphemen labh- 
und $\angle s / 1 \frac{2}{a} s^{w}$, die als konventionell betrachtet werden. Das Morphem pāk'-,pāg'- fällt wiederum durch seine nahezu vollständige Metathese der imitativen kap- und ghabh- auf; in seinem Aufsatz "Fangen, Finger, fün" hat schon W.Oehl darauf aufmerksam gemacht. Und das $\mathrm{CH}$ im slavischen chop-, das seiner Form nach dem indoeuropäischen ghabh- am nächsten steht, hat seinen expressiven Charakter offenbar dem Umstand zu verdanken, dass es als Reibelaut im Anlaut stark ingressiv artikuliert werden kann wie das fir einen Verschlusslaut nur im Auslaut möglich ist und so das Schnappen in seiner Richtung auf den Rachen hin am besten nachahmt. Alles das bezieht sich nun in unserer serie auf das schnelle Zugreifen mit der Hand. Um das Bild zu vervollständigen, führen wir noch zwei weitere Serien kurz an. In der Serie TAKE /B 11.13; Ling. VIII/1, 23/ besitzen 4 der 14 primären indoeuropäischen Wurzelmorpheme - ein ungezähltes Wurzelmorphem ist einzelsprachlich - die A-Charakteristik, also von neuem das Doppelte vom Durchschnittlichen. Da die Morpheme ausser ag'- zu Gr. agreō die gleichen wie in der vorigen Serie sind, brauchen wir nicht in die Einzelheiten einzugehen. Die dritte zu erwähnende serie ist KEEP, RETAIN /B 11.17/. Mit dem Verhältnis der A-Charakteristiken zur Gesamtzahl der untereinander unverwandten Synonyme, das hier $5 \mathrm{zu} 14$ beträgt, scheint die Serie, mit einem unerklärten Synonym 15 Einheiten zählend, noch stärker ikonenhaft bedingt $\mathrm{zu}$ sein als die Erstangefuhrten. So haben wir kar- zu krateō, Ngr. kratō, ghabh- zu Ir. congaibim, katoder k'at- zu Cymr. cadw, past- zu Got. gafastan und g'ăb- zu Me. kepe, Ne. Keep. Das Auftreten des Morphems $\mathrm{g}, \frac{\mathrm{a}}{\mathrm{a}} \mathrm{b}-\mathrm{neben}$ dem anerkannt imitativen ghabh- ist besonders interessant, weil seine Struktur mit unaspirierter Media im Anlaut wie im Auslaut in der rekonstruierten Ursprache ganz ungewöhnlich ist; die gleiche Erscheinung haben wir schon in der serie "ansehen" bei dem Wurzelmosphem stilp-stilb- beobachten können. Doch wenden wir uns von den oralen und manuelien Ikonen des Schnappens und Greifens weg und einer Gruppe andersartiger. A-Charakteristiken zu, die sich von den bisher behandelten formell und semantisch stark 
abheben. Die A-Morpheme, denen wir begegnet sind, hatten durchgehend die Form: Konsonant, Vokal, Konsonant /CVC/ und der AVokal war fast ohne Ausnahme als kurz oder als von unentschiedener Qualität bezeichnet. Die A-Charakteristiken, die wir uns nun ansehen wollen, beziehen sich jedoch ausschliesslich auf ein indoeuropäisches langes $A$, sei es im Wurzelmorphem oder in der Wurzelerweiterung, das gewöhnlich in der Form: Konsonant oder konsonantencluster plus Vokal /CV,CCV,CCCV/ auftritt. Bei der statistischen Untersuchung von Bucks Wörterbuch hat es sich herausgestellt, dass die Charakteristik des langen $A$ in den primären Wurzelmorphemen besonders deutlich in Korrelation zum Sinngehalt des "Flachen" steht. Die Serie FIAT/B 12.71; Iing. VII/2, 14I/ enthält 10 Synonyme, die alle etymologisch erklärt sind. Da die dazugehörigen Wurzelmorpheme das $\bar{A}$ dreimal aufweisen, während es im Durchschnitt kaum einmal in einer Serie durchschnittlichen Umfangs zu erwarten wäre, ist geniggend Grund zur Vermutung einer ikonenhaften Einwirkung gegeben. Zunächst erscheint das $\bar{A}$ im griechischen synonym: platus, welches auf eine erweiterte Wurzelform plā- zurickgeht, dann im Lat. lātus, das einem erweiterten stlā- enstammt, und endlich im Cymr. gwastad, welches eine präfigierte Form des einfachen wurzelmorphems stā- darstellt. Nun werden alle diese Synonyme mit den dazugehörigen Wurzelformen als unzweifelhaft konventionelle Sprachzeichen betrachtet, ein Umstand, der uns bei den vorangegangen Analysen, wo wir uns jederzeit auf anerkannte Ikonen berufen konnten, noch nicht begegnet ist. Was spricht ausser den uberzähligen A-Charakteristiken fur unsere Annahme eines Ikonismus des "Flachen"? Vor allem sprechen daflir zusätzliche Formcharakteristiken, ungewöhnliche Konsonantencluster im Anlaut und expressive Konsonantengemination, sei es in den A-synonymen selbst, sei es in verwandten wörterm. Geminiert ist das Vlat. *plattus, das wahrscheinlich aus dem Griechischen entlehnt worden und in die romanischen Sprachen ubergegangen ist, und geminiert ist Lat. stlatta "breites Schiff", das noch den vollen Anlautskonsonantismus bewahrt hat, welcher in: lātus reduziert 
auftritt. Dieser Anlautskonsonantismus stl- ist im Lateinischen wie auch in anderen indoeuropäischen sprachen sehr selten und offenbar archaisch; so haben wir lateinisch: stlocus, eine ältere Form von locus "Ort", stlembus "schwerfällig, langsam" und stloppus "Gefäss mit rundem Hals" und "schallende Ohrfeige". Doch, während stlocus und stlembus als konventionelle Sprachzeichen dem gleichen Wurzelmorphem stel-, dem: lätus und stlatte angehören, zugeteilt werden, wird: stloppus als onomatopöie aus der Familie ausgeschlossen und als ein vollkomen isoliertes Wort behandelt, trotzdem es formell eben dieselbe struktur aufweist wie die vorangegangenen Worter. Hier stossen wir an ein prinzipielles Zuordnungsprinzip der Etymologen, die im Einklang mit de Saussures Bewertung der Onomatopöien,jeden Zusammenhang zwischen ihnen und den als konventionell betrachteten Sprachzeichen verneinen Einstweilen wollen wir dies nur feststellen. Denn auch in der Nachbarschaft des griechischen Synonyms: platus und der von ihm entlehnten Synonyme treffen wir gleichartige Begleiterscheinungen an. Die slavischen Synonyme Aksl. ploskŭ, Russ. ploskij usw., welche demselben erweiterten Wurzelmorphem entstammen wie Gr. plaths, haben in Slov. ploskati "schlagen/so dass es patscht/", "in die Hände klatschen", "patschen"; "herumpatschen" eine formell genau entsprechende Onomatopöie neben sich; in der Ablautstufe mit E: Russ. pleskst' und den parallelen E-Formen in anderen slavischen Sprachen, das Slowenische einbegriffen, tritt wiederum die gleiche Bedeutung "klatschend, patschend, schlagen" auf. Auch in diesem Fall lehnt die traditionelle etymologische Wissenschaft jede Verbindung mit den Wörtern für "flach" vom Wurzelmorphem plā- ab, trotzdem unter einem homophonem und etymologisch gleichgesetztem plä- Wörter aus alten, toten Sprachen, z.B. Ir. lessaim und Ags. flōcan, mit der identischen Bedeutung "heftig, klatschend schlagen" zu finden sind. Dieser letzte Umstand zeigt, dass das wesentliche Kriterium für die Bestimmung der Onomatopöien, nämlich die Schallbedeutung, nicht ganz konsequent angewendet wird, offenbar im Verhältnis damit, wie alt die Zeugnisse von einem solchem Wort sind. Die 
Liste der Inkonsequenzen wäre lang; ich brauche nur auf Ai. vakti, vívakti zum Wurzelmorphem wek $^{\text {w }}$ - und Lat. fārĩ zum Wurzelmorphem bhä- aus der. Synonymenreihe für "sprechen", hinzuweisen. Niemandem ist es je eingefallen, diese wörter und wurzelmorpheme als onomatopoetisch zu bezeichnen, wie das für viele Synonyme in der gleichen Reihe zutrifft. Andererseits ist von der etymologischen Wissenschaft kaum der Versuch untemommen worden, die Beziehung der als onomatopoetisch angenommenen sprachzeichen zu den bezeichneten akustischen Phänomenen systematisch zu untersuchen; die pauschale Feststellung, dass in der Bezeichnung des gleichen Schalles die Sprachen verschiedene Wege gehen, genügt nicht. Aus unserer Erkenntnis des nichtonomatopoetischen Ikonismus,heraus, der mit dem konventionellen Sprachschatz eng verbunden ist, können wir auch zwischen konventionellen sprachzeichen und Onomatopöien keine prinzipielle trennende Grenze anerkennen; die ausstehende systematische Untersuchung aller Sprachzeichen, die sich auf hörbare Phänomene beziehen, wirde gewiss diese Grenze, mag sie wohl einer alten Tradition entsprechen, völlig niederreissen. Im Fall von Aksl. ploskŭ usw. ist es also nicht nur zulässig, sondern sogar geraten, den Vergleich mit den onomatopoetischen: ploskati, pleskati anzustellen, weil uns daraus zusätzIiche Informationen zufliessen. Beide genannte Zeitwörter haben im Slowenischen Interjektionen: plosk!, plesk!, mit der Bedeutung. "platsch! patsch!" neben sich, die sich unter anderem auf den Schall einer Ohrfeige beziehen. Die Ohrfeige ist nun ein Schlag mit der flachen Hand und die semantische Beziehung zum altkirchenslavischen: ploskŭ, slowenischen: plosk "flach". liegt offen zu Tage. Wir müssen uns erinnerm, dass wir auch in Lat. stloppus der gleichen Bedeutung "schallende Ohrfeige" begegnet sind, was einen klaren Parallelismus im Bedeutungsverhältnis "Schlag mit der flachen Hand" - "flach" darstellt. Und wir haben es bisher nicht erwännt - eben dasselbe Bedeutungsverhältnis existiert im konventionellen Sprachbereich zwischen Gr. platagé "das Klatschen", platagēo "klatschend schlagen" und: platus! Wir ersehen aus all diesem,dass die Vermutung des Ikonismus in Gr. platus und in Lat. lātus, die sich primär auf 
die ungewöhnliche Wiederholung der A-Charakteristik und andere rein formale Charakteristiken stutzte, durch die Anwesenheit form- und bedeutungsverwandter onomatopäen ausdrulcklich bestätigt wird. Irre Anwesenheit ermächtigt uns sogar zur weiteren Vermutung, dass wir bein Ikonismus des "Flachen" mit dem Übergang von onomatopoetischen Ikonen $\mathrm{zu}$ nichtonomatopoetischen $\mathrm{zu}$ tun haben. Ehe wir an anderen Serien mit uberzahliger $\mathbb{A}$-Charakteristik die Entstehung nichtonomatopoetischer Ikonen weiterverfolgen, fuhren wir noch einen diesbezüglichen Zusatz zu unserer Grundserie an. Das lettische, von Buck ubersehene Synonym: blaks "flach" steht allem Anschein nach mit dem Iit. blakštas "Schlag mit der flachen Hand", blakštuti "damit oder mit einem anderen Ding ähnlich schlagen" in Verbindung. Nun noch zwei Serien aus einer grösseren Gruppe, die wir der Serie fur "flach" als gleichartig anfugen können. Die Serie PLACE /B 12.11; Ling. VI,34/ enthält von 16 etymologisch erklärten Synonymen - eines ist unerklärt-wiederum 3 mit einer $\mathbb{A}$-Charakteristik im Wurzelmorphem statt eines einzigen. Es sind dies Fr. place tber lat. platea, Gr. platela zum erweiterten wurzelmorphem plā-, Got. staths, Ahd. stat zu stāund Av. gātu- zu gwā-. Das französische Synonym ist uber die lateinische Form direkt aus dem griechischen Adjektiv: platus abgeleitet und braucht keine weitere Erläuterung. Wenn wir jedoch bei der Zusammenstellung des germanischen und des iranischen Synonyms auf das Reimwurzelpaar furr "stehen" und "gehen" aufmerissam gemacht werden, steight ungewollt der Verdacht auf, ob vielleicht nicht auch hier spuren eines alten $\bar{A}$-Ikonismus vorhander seien. Doch fehlen hier die Begleiterscheinungen eines ungewöhnlichen bzw. expressiven Konsonantismus und nahestehender Onomatopöien, so dass wir die gestellte Frage offen lassen mussen. Die Bekräftigung des Verdachtes kommt von einer ganz anderen Seite. Das griechische Synonym: topos hat nämlich eine weit verbreitete slavische Onomatopöienfamilie zur Seite stehen, die Z.B.in Russ. tepti "schlagen", aber auch ablautend in topat, "stampfen, stapfen" auftritt, und ausserdem in Lit. tàpštereti "klapsen", tapšt! "klatsch! klapps!"und Lett. tapat "stampfen, stapfen" 
bezeugt ist. Das Bedeutungsverhältnis von: topos zu dieser onomatopoetischen Familie zeigt einen verbluffenden Parallelismus zum Bedeutungsverhältnis von: platks - platageō, ploskŭ - ploskati, Iātus - stloppus. Aber nicht nur das. Auch die Bedeutungen "stampfen" und "stapfen" d.i. "mit dem ganzen Fuss stehend oder gehend schwer auftreten" haben in den Ablegerm der Wurzelformen plā- und stlā- z.B. in Iat. planta "Fussohle" plancus "plattfüssig" sowie wahrscheinlich in dem schon erwähnten: stlembus "langsam, schwerfällig" genaue Parallelen. Auf einmal wird klar, dass das $I$ in den Wurzelmorphemen für "stehen" und "gehen" durch die Bedeutungen "stampfen" und "stapfen" am besten erkärbar sei, und dass das lateinische: locus, wenn wir es zusammen mit stiembus von der erweiterten wurzel stia- statt von der unerweiterten stel- ableiten, eben dieselbe Bedeutungsentwicklung aufweist wie Got. staths, Av. gātu- und Gr. topos. Eine weitere Serie, in der wir den hier beschriebenen semantischen und formalen Zusammenhang antreffen können, ist die serie BOARD /B 9.52;Ling. VI,27/. Unter 11 unabhängigen Synonymen, von denen 10 etymologisch erklärt sind, befinden sich 2 mit einer alten A-Charakteristik: Fr. planche von Spätlat. planca zu plā- und Ir., Nir. clär zu klä-, der Erweiterung einer Grundform kel-. Die Bedeutungen der Wörter, die unter kel-,klea- etymologisch vereinigt sind, werden mit der allgemeinen Bezeichnung "schlagen, hauen" umschrieben; für un's ist es jedoch an wichstigsten festzustellen, dass wir von neuem neben wörtern mit der "Bedeutung "Brett" und "Tafel" auf Ausdricke fir "Ohrfeige" und "treten" in Gr. kblaphos und: koletran stossen. Diese wörter weisen zwar, genau genommen, kein langes $\mathbb{A}$ auf, da sie eine zweisilbige Erweiterung der Grundform keldarstellen, doch gehören sie zur Schwundstufe des gleichen Ablautsystems und fallen offenbar in das Bedeutungsfeld des "filachen Schlagens" usw. Die semantische Ableitung der durch die A-Charakteristik bezeichneten Ikonen fir "flach" und "etwas Flaches" aus onomatopoetischen bzw. onomatopöeienglaichen sprachzeichen für "klatschend schlagen" und "stampfen, stapfen" scheint somit genügend erwiesen zu sein; es fehlt nur noch die semiotische 
Deutung des ganzen Zeichenprozesses. Was hat der Vokalismus des langen I mit dem Formphänomen des "Flachen" und dem akustischen Phänomen des "klatschenden Schlagens und Auftretens" zu tun? Obgleich bei der semiotischen Deutung von diesem akustischen Phänomen auszugehen ist, kann das A keine Schalinachahmung sein; eine elementare Kenntnis der Akustik schliesst jede Ahnlichkeit zwischen dem Schall des $\bar{A}$ und dem Schall des genannten Schlagens aus. Den Schlüssel zur Lösung des Problems gibt wiederum die Artikulation des $\mathbb{A}$. Wir haben gleich am Anfang unserer Beschaftigung mit diesartigen Ikonen darauf aufmeriksm gemacht, dass es sich bei unserer Charakteristik um ein langen $\bar{A}$ im Auslaut der silbe handelt. Wenn wir nun im den wurzelmorphemen mit kurzem Vokal in Mittelstellung und konsonantischem Auslaut die Nachahmung des zusammenschlagenden Mundes festgestellt haben, so durfen wir bei wurzelmorphemen mit $\bar{A}$ im Auslaut auf die Nachahmung des sich öffnenden und offenen Mundes schliessen. Doch der systematische Utbergang in Sprachzeichen mit der Bedeutung "flach" legt uns noch eine zusätzliche, entscheidende Erklärung

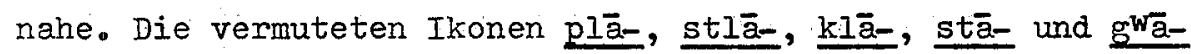
ahmen in erster Linie die Zungenartikulation des $\mathrm{A}$ nach. In ruhender stellung schmiegt sich die Zunge mit ihrem Vorderteil an den Gaumen und bei der Artikulation des A muss sie sich zum Mundboden hin bewegen, was bei intensiver Artikulation einem Zungenschlag gleichkommt; bei vollendeter Artikulation liegt sie flach auf dem Mundboden. Diese Erklärung wird uberzeugend damit gestiutz, dass teils in den $\mathbb{A}$-Wurzelmorphemen teils in Wurzelmorphemen mit neutralem $\mathrm{E} / \mathrm{O} \mathrm{Vokal}$ in allen hier behandelten Serien parallel ein uberzahliges I aufritt; so hat die Serie FIAT $3 \mathrm{~L}$ in 10 Morphemen, die Serie PIACE 3 in 16 Morphemen und die Serie BOARD sogar 5 in 16 Morphemen, was das zweifache bis dreifache des Durchschnittlichen beträgt. Das I ist nun das einzige Phonem im indoeuropäischen Phonembestand, das bei seiner Artikulation die Wahrmehmung der sich bewegenden Luft an den Zungenseiten und an dem Mundboden voraussetzt. Darum eignet es sich am besten von allen Konsonanten für die Nachahmung des 
Zungenschlags; es ist eine konsonantische Nachahmungsdublette des auslautenden $\mathrm{A}$. Wie im Fall des letzteren ist auch seine Nachahmungsrolle wesentlich artikulatorisch, doch diffen wir neben bei auch eine Schallnachahmung des Zungenschlags annehmen, denn nur um eine solche kann es sich hier wie auch bei anderen konsonantischen Elementen der ikonenhaften Wurzelmorpheme fur "klatschendes Schlagen" und "flach" handeln. Überall, wo wir manuellen oder pedalen Ikonen mit einer $\bar{A}$ - oder I-Charakteristik begegnen, geht es entweder um mehr oder weniger bewusste ưbertragungen von halb onomatopoetisch gefärbten artikulatorischen oralen Ikonen oder um primäre somatische Ikonen, die direkt aus der unbewussten Perzeption des neuromotorischen Zusammenhanges zwischen ähnlichen Körperbewegungen gebildet sind, welche jedoch in ihrer Struktur gleicherweise auf die artikulatorische Nachahmung der Körperbewegungen zurückgehen. Untersuchungen uber etwaige Synergie zwischen den Bewegungen der Sprechorgane, der Hände und Fisse wïrden eine grössere Klarheit über die gegenseitige Bedingtheit dieser Bewegungen bringen. Jedenfalls sind bei den Wurzelmorphemen unserer Serien auch orale Bedeutungen ausdricklich bezeugt, so z.B. in Russ. kolotit, zu kel-, das ausser "schlagen, klopfen" auch "plappern" bedeutet, oder in Tschech. pleskati, pleštiti, neben dem homophonen plosky zu plä-, das unter anderem die Bedeutung "schwatzen" aufweist. Nun sind wir aber zum Ausgangspunkt unserer Ausfuhrungen uber die nichtonomatopoetische ikonische Synonymie zurickgekehrt. Wir haben sie zum leichteren Verständnis der angewandten quantitativqualitativen, realitatsvergleichenden Methode mit der Analyse der onomatopoetisch bedingten Synonymenreihe SPEAK, TAIK begonnen. Dabei haben wir festgestellt, dass in der serie eine stark uberzählige I-Charakteristik erscheint und dass sowohl bei den anerkannten Onomatopöien wie bei den als konventionell betrachteten Wörterm fast regelmässig I als zweiter Konsonant im Wurzelmorphem auftritt. Diese Erscheinung ist damals unerklärt geblieben und uberhaupt ist keine semiotische Deutung der formalen Morphemcharakteristiken versucht worden. Wir können im Licht unserer 
gesamten Erkenntnisse jetzt beides nachholen. Die Serie fur "sprechen" und die Serien fur "flach" und verwandte Bedeutungen haben namlich eine im wesentlichen gleiche Morphem- und Phonemstruktur.Auch in den Serien fur "flach" usw. zeigen 8 von den 11 auftretenden I-Charakteristiken das $I$ an zweiter Konsonantenstelle. Andererseits hat die Serie fur "sprechen" zusätzlich zu seinen uberzähligen wurzelmorphemen mit $L$ auch uberzählige

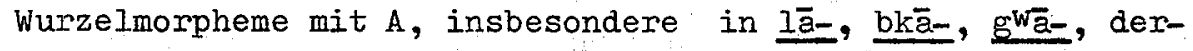
jenigen mit langem $\bar{A}$. Neben lea- zu Gr. laleo, plab- zu Ir. labrur und gal- zu Aksl. glagolati erscheinen nämlich bhā- zu Lat. färī und giwa- uber Lat. conventus zu Rum. Cuvinta. Doch ist das Wurzelmorphem plab- unter ihnen besonders interessant. Es vereinigt ausdricklich die Bedeutungen "sprechen" und "schlagen" und zeigt auch die vermittelnde Bedeutung "schwatzen" auf, indem es dem Ir. labrur das niederdeutsche flappen "schlagen, klatschen, schwatzen" und das neuenglische: flap entgegenstellt. Die semantische Entwicklung von "schwatzen" zu "sprechen" haben wir schon bei den valgärlateinischen: fābulāre und parabolāre behandelt; sie ist ein sociolinguistisches Phänomen, verbunden mit gesellschaftlichen Umschichtungen. Das Nebeneinander der Bedeutungen "schlagen" und "schwatzen" ist jedoch gerade diejenige psycholinguistische Erscheinung, die wir am Beispiel des Russ. kolotit, zu kel- als artikulatorische Nachnahmung der Körperbewegung, die sowohl orale wie manuelle Bedeutungen umfasst, gedeutet haben. Der demonstrative wert des Wurzelmorphems plabund seiner Bedeutungen besteht darin, dass es als anerkanntes altes indoeuropäische schallwurzelmorphem uberzeugender als das für konventionell gehaltene russische kolotit' - oder das nur dem Baltoslavischen zugerechnete tschechische schallwort: pleskati, pleštiti - die Gesetzlichkeit einer bestimmten Iautstruktur für "klatschendes Schlagen", sowohl mit der Zunge wie mit der Hand, darstellt. Die Form des Wurzelmorphems enthält vor allem die I-Charakteristik mit I an zweiter Konsonantenstelle, doch sie ist noch weiter dadurch charakterisiert, dass an erster Konsonantenstelle ein Verschlusslaut steht. Die dritte und 
wichtigste Charakteristik besteht endlich darin, dass der Verschlusslaut und $I$ unmittelbar im $A_{n l a u t}$ aufeinanderfolgen. Diese letzte Lautkombination tritt nun auch in einer grossen Reihe neusprachlicher Interjektionen, Zeit- und Hauptwörter auf - mit den deutschen: klatsch! klapp! klack! platsch! angefangen - , welche mit den Bedeutungen "klatschend schlagen, stapfen oder fallen" ein intensives Gefuhl fur die richtige Wiedergabe der bezeichneten Bewegungen und Schalle verbinden. Ein solches Gefunl ist genau so stark in den schon zitierten slowenischen Interjektionen plesk! plosk!, denen sic hein ähnliches: tlesk! mit dem im Deutschen fehlenden tl-Anlaut zugesellt. Den Grund für das Gefuhl sehe ich in dem Umstand, dass die Nachahmungskraft des I für den Zungenschlag durch den / unmittelbar/ vorhergehenden Verschlusslaut insoweit verstärkt wird, als der Verschlusslaut im Anlaut das öffnen des Mundes von oben nach unten besser darstellt als der spirantenähnliche I-Iaut. Deswegen erweckt auch ein Spirant vor dem I kein ebensolches Gefuhl des Ikonismus. Die typologische Untersuchung der Ionomatopoien in den sprachen der welt auf ihren semantischen Gehalt hin könnte zur lösung des Problems verschiedener Varianten dieser ikonischen Phonemstruktur gewiss viel beitragen. Dabei missten die lateralen Affrikaten und die I-flaps, wo sie bestehen, in die Untersuchung miteinbezogen werden. Schon jetzt steht fest, dass die Gesetzlichkeit des Lautmodells des imitativen Wurzelmorphems plab- sowohl in imitativen wie als konventionell betrachteten indoeuropäischen wurzelmorphemen bezeugt ist, nichts destoweniger aber auch in den als einzelsprachlich angenommenen und als selbständige Schallnachahmungen kategorisierten wörterm, welche dem behandelten Sinnbereich des "klatschenden Schlagens" usw. angehören. Ausser eines solchen Parallelismus bei plab- selbst, das zusammen mit dem slawischen imitativen: plosk dem scheinbar konventionellen plä- in $\mathrm{Gr}$. platagé, platageó angereint werden könnte, und ausser des Nebeneinaders der lateinischen: stlocus "Ort" - stloppus "Ohrfeige", futhren wir noch ein paar andere Beispiele dieser semantischen 
und lautlichen Übereinstimmung so verschieden kategorisierter sprachlicher Zeichen an. So stossen wir unter der wurzelform bhlë́d-, die man als Erweiterung des konventionellen wurzelmorphems bhel- auffasst, auf ein Gr. phledōn "Schwätzer", phledón "Geschwätz"; auf eine gleiche indoeuropäische Form könnten die deutschen Wörter: platzen, platschen, plätschem zuruckgeführt werden, wenn man sie nicht als "wohl sicher jüngere Schallbildungen" vom Vergleich mit den griechischen wörtern ausschliessen wïrde. Unter einem als konventionell betrachteten Wurzelmorphem bhlag'- finden wir Lat. flagrum, flagellum "Geissel, Peitsche" neben An. blekkja "schlagen"; das Lett. blaks "flach" mit dem lit. bl5kštas "Schlag mit der flachen Hand", die sich nur im Fehlen der Palatalisation beim Endkonsonant von ihnen unterscheiden, werden hingegen als isolierte baltische wortbildungen angenommen. Die Grundform des An. klaka "schwätzen" und "zwitschern", des Ne. clack "plaudern" und "klappern", des Mhd. klak "Klecks, Fleck" usw. wird als ein indoeuropäisches onomatopoetisches glag- rekonstruiert und unter eine germanische Grundform: *klat-, *klap- werden weiter Nhd. Klatz "Schmutzfleck", Aha. Klapf "Geschwätz, Knall, Schlag, Stoss" angereiht. Diesen in J. Pokomys indoeuropäischem etymologischem Wörterbuch solcherweise erklärten wörterm durfen wir wohl die schon erwähnten neuhochdeutschen Interjektionen: klack! klatsch! klapp! mit den dazugehörigen Zeit- und Hauptwörten hinzufiugen. Andererseits werden jedoch die gleichen wörter als erweiterte Formen des konventionellen Wurzelmorphems gel- "ballen, sich ballen usw." angefuhrt /P. 358, 359, 360/; es wird nur die expressive Konsonantengemination dabei betont, ohne irgendeine Erklärung der Ursache zu geben. Auf das russische: kolotit' "schlagen, klopfen" und "plappern" zum konventionellen wurzelmorphem kel- haben wir schon hingewiesen; unter dem gleichen kel- begegnen wir dem griechischen: k6laphos "Ohrfeige"; das homeophone slawische: klep-, klop-, welches z.B. im Slowenischen mit den Synonymen: klepetati "schwätzen", klofuta "Ohrfeige" vertreten ist, wird hingegen als selbständiges nur dem Baltischen 
und Slavischen gemeinsames imitatives Morphem erklärt. Neben dem Wurzelmorphem tolk $\mathrm{K}^{\mathrm{W}}$, das als nichtimitativ gilt und das in seiner lateinischen Form *toqui, loqui die unmittelbare lautfolge tI- aufweist, haben wir ein gleich kategorisiertes telekmit der Bedeutung "schlagen", z.B. in Russ. toloč', das nur in der fehlenden Labialisierung des Endkonsonanten von dem vorigen abweicht; es reihen sich an teleg'h- mit dem litauischen Bedeutungspaar: teležyti "gewaltig durchprügeln" und talažutoti "schwatzen", telp-, das wegen Russ. tolpa "Haufe, Schar" auch auf eine Grundbedetung "schlagen" hinweist, und endlich telmit der Grundbedutung "flach", dem wir in der Serie BOARD bei Lat. tabula begegmen. Daruber hinaus aber werden alle diese als konventionell betrachtete primären indoeuropäischen Wurzelmorpheme von einer Menge als onomatopöisch deklarierter wörter in verschiedenen slavischen Sprachen begleitet $z \cdot \dot{B}$. in Russ. telepat' sja "sich herumtreiben", eigentlich: "sich herumschlagen", teleljachnut, "Reissaus nehmen", das mit dem Ukr. telechnuty "heftig schlagen" verbunden wird, teleljakat? "schwatzen" usw. Im Ukrainischen haben wir unter anderem noch: telepnuty "eine Ohrfeige geben" mit der dazugehörigen Interjektion: telep! "klatsch", im Slowenischen: telebati "plump schlagen /auf die Trommel/" und die schon erwähnte Interjektion tlesk! "klatsch!" neben dem Verbum: tleskniti "einen schallenden Schlag versetzen", "klatschen" und "mit der Zunge schnalzen". Eine eng verwandte orale Bedeutung tritt in dem gleichlautenden tschechichen: tleskati "/schmatzend/ kauen" auf, während in tlapa "Tatze" die Grundvorstellung "flach" erscheint. Hier wie uberhaupt sind die Beispiele des morphosemantischen Parallelismus, die wir vom Morphem plab- ausgehend angefuhrt haben, lange nicht erschöpft; leider fehlen bei Buck die Serien fur "Ohrfeige, klatschend schlagen, stapfen, schwatzen" und noch manche andere, die in unseren Zusammenhang gehören. Doch die Beispiele genügen, um ein einheitlich strukturiertes formales und semantisches system von lexikalischen Einheiten aufuzeigen, das wir nach P.Guiraud ein morphosemantisches Feld nennen können. Im Laufe unserer 
Analysen sind wir mehrmals an ein solches Feld gestossen. Das Besondere in diesem Fall ist jedoch, das es anscheinend aus drei, aus verschiedenen Quellen herrihrenden, heterogenen Bestandteilen besteht. Aber gerade das gibt uns die Möglichkeit, das Problem artikulatorischer Ikonen noch einmal mit neuer Fragestellung aufzurollen. Existiert nicht ein fundamentaler Unterschied in der semantischen Präzisierung bei alten, toten sprachzeichen aus Zeiten, wo noch keine Worterbucher und keine systematischen Übersetzungen vorhanden waren, und bei den lebendigen Wörtern der modernen Sprachen, deren Sinngehalt jeden Tag kontroliert werden kann und in zahlreichen ein- und mehrsprachigen Wörterbuchem festgehalten wird? Ist nicht gerade dieser Unterschied in hohem Grade schuld daran, dass man die alten wörter bzw. ihre Wurzelelemente fur "schlagen" usw. als konventionelle Sprachzeichen auffasst und ihre schallbedeutung als nicht vorhanden beiseite schiebt, wo doch diese in ihren modermen synonymen deutlich erkennbar ist und auch erkannt wird? oder sollten vielleich die wörter für "schallend schlagen" ein nur den modermen Sprachen eigenes Phänomen sein? Ich glaube, dass wir eine solche Alternative als absurd verwerfen und eine Gleichstellung alteronomatopöien für orale Laute und der anscheinend konventionellen alten wörter fur "schlagen" und andere mit Geräuschen verbundene körperbewegungen annehmen müssen, besonders dort, wo sie mit dem Lautmodell der modermen Synonyme, wie in den angeführten Beispielen, genau übereinstimmen. Das ist eine der Schlussfolgerungen, die sich aufdrängen. Die andere betrifft das Verhältnis zwischen alten und jungen Onomatopöien. Diese beiden Kategorien voneinander prinzipiell zu lösen ist nur möglich, wenn man an die Neuerschaffung onomatopoetischer Sprachzeichen in modermer oder neuerer Zeit aus einem sprachlichen Nichts glaubt, was tatsächlich in den etymologischen wörterbüchern vorausgesetzt wird. Auch diese Ansicht ist beim näheren Betrachten höchst unwahrscheinlich. Historisch ist ein solches Entstehen ursprachlicher Schöpfungen bei voll entwickeltem Sprachzustand nirgends bewiesen; wenn we sich nicht in solchen Fallen um 
direkte Ableger alter Wurzelmorpheme handelt, geht es um zwar sekundëre, doch an jene angelehnte Bildungen entweder durch Umformung, Undeutung oder Entlehnung existierender, unverwandter Sprachzeichen. Das ist die zweite Schlussfolgerung. Denn wie könnte man sonst das gleiche Lautmodell und den gleichen semantischen Inhalt bei alten und neuen onomatopoetischen Synonymen erklären? Die dritte Schlussfolgerung bezieht sich noch einmal auf das Wesen der onomatopöien und das Wesen der artikulatorischen Ikonen sowie auf ihr gegenseitiges Verhältnis. Der subjektive Eindruck der Onomatopöien des Schlagens und anderer körperlicher Bewegungen, als seien sie eine akustische Wiedergabe der dabei entstehenden Geräusche, ist ganz gewiss trigerisch, nicht nur, was die vokalischen, sonderm auch, was die konsonantischen wortelemente betrifft. Die vokalische Charakteristik in ihnen ist rein artikularisch-ikonisch, die konsonantischen Charakteristiken hingegen haben eine ikonische Doppelnatur: einerseits stellen auch sie den artikulatorischen Ikonismus des Zungenschlags dar, andererseits jedoch dessen auditive Nachahmung. Nur mit einer solchen Beschränkung können also die Onomatopöien fur "schlagen". usw. als wirkliche Onomatopöien gedeutet werden. Die subjektive Täuschung über den akustischen Wert der Lautelemente ist ein ausdricklicher Hinweis auf den neurophysiologischen Zusammenhang der Körperbewegungen. Dieser subjektive Gehörseindruck der artikulatorisch-auditiven Ikonen des /flachen/Zungenschlags wird dann auch auf die Formikonen für "flach" und einzelne flache Phänomene ubertragen, wo er jedoch im Iaufe der Zeit friher verblasst als bei den Tätigkeitsikonen. Noch eine letzte Bemerikung drängt sich auf. Der Ikonismus, den wir analysiert haben, bezieht sich ausser auf den $\bar{A}$-Vokal nur auf die ersten zwei Konsonanten der angefuhrten Sprachzeichen, höchstens auf drei, die vor dem Vokal im Anlaut stehen. Die Analyse der unbericksichtigten dritten oder auch vierten Konsonanten, welche in den besprochenen Sprachzeichen nach dem Vokal im Auslaut auftreten, kann zwar hier im eirzelnen nicht durchgeführt werden, es genulgt die Grundlinie der semiotischen Deutung 
aufzuzeigen. Beim dritten bzw. dritten und vierten Konsonanten geht es entweder um Intensivierung der Ikonen des Zungenschlags durch endosyllabische Reduplikation oder durch eine dem Anlaut gleichwertige Nachahmung, wie z.B. in den deutschen: klack! und klatsch!, oder es handelt sich um die Kombination zweier verschiedener Ikonen, der Ikone des Zungenschlags und der Ikone des zusammenklappenden Mundes, wie $z . B$. in dem deutschen: klapp! Weil der Labial im Auslaut am besten das Zusammenklappen des Mundes wiederzugeben scheint, durfen wir jedoch nicht diese Nachahmungsfuntion generell als seine einzige ikonische Funktion festlegen. Der Labial in Auslautstellung kann - ungeachtet der Etymologie - auch als:Metathese aufgefasst werden, was in einzelnen Falle aus dem Semantismus hervorzugehen scheint, wie z.B. in Słow. lop! "patsch!", lopati "mit einem flachen Gegenstande schlagen". Oder sollen wir annahmen - auch das ist möglich dass das Zusammenschlagen der Kiefern und der Lippen ähnlich mit der Bewegung des Schlagens verbunden ist wie der Zungenschlag? Eine systematische neurophysiologische Untersuchung in dieser Richtung wäre gewiss nicht überflüssig.

Ich muss schliessen. Die bei dieser Gelegenheit analysierten Arten der artikulatorischen Ikonen stellen nur eine Einleitung, bei weitem keine vollständige Übersicht des artikulatorischen Ikonismus in der sprache dar. Das erhellt schon daraus, dass ich die zwei im Abstrakten vorgefuhrten Ikonen fur "rauh" mit der R-Charakteristik und für "Loch" mit der U-Charakteristik unberúcksichtigt lassen musste. Indem die letzte der Synonymenreihe fïr "Mund" anzureihen ist, handelt es sich in der serie fur "rauh" nur um eine der zahlreichen Varianten der R-Charakteristik. Die Einreihung dieser und noch anderer artikulatorischer Ikonenarten in ein möglichst vollständiges Modell des artikulatorischen, mit dem menschlichen Körper verbundenen Ikonismus soll einer kïnftigen Gelegenheit überlassen werden. 
Božo Vodušek

(1905-1978)

linguistica je spet izgubila svojega sodelavca. Božo Vodušek je združeval v sebi več nagnjenj: bil je pravnik, študiral je romanistiko, zadnja desetletja svojega življenja pa se je ukvarjal z jezikoslovjem. In bil je tudi pesnik. Morda je prav ta nenavadna kombinacija, poet - znanstvenik, narekovala njegovo pot $v$ jezikoslovju. Linguistica je v letih 1964-71 objavila skrbno nabrano gradivo iz indoevropskih jezikov; in to je bilo $\mathrm{v}$ dobri meri podlaga $\mathrm{za}$ študijo, $\mathrm{ki}$ jo tu objavljamo. Gradivu je postavil na začetek kot motto tezo učitelja svojih mladostnih študij v Parizu, Antoina Meilleta: "Le fait que les memes notions sont exprimées dans les diverses langues humaines par des sons infiniment divers et que le sens attribue aux mots varie sans que les sons $y$ soient intéressés ou que, inversement, la prononciation des mots varie sans que le sens $y$ soit intéresse suffit à montrer qu'il est inutile de rien chercher de ce cote."

Vodušek je mislii čisto drugače: vse svoje moči je posvetil nalogi, da na podlagi indoevropskega gradiva dokaže, da najdemo genealoško nerazložljivo povezanost tudi zunaj ozkih meja tradicionalno pojmovane onomatopoetičnosti ali nejasno določene ekspresivnosti. opravil je znanstveno zelo rigorozno analizo indoevropskih korenov: statistika je pokazala, da gre za očitno nenaključno razdelitev fonemskih karakteristik. Relativna frekvenca neke glasovne karakteristike ni naključna in ker je pogostna, jo je težko pripisati kakemu drugemu vzroku kot notranji zvezi med pomenom in glasom. Saussurov jezikovni znak, se pravi povezava med označenim in označujočim, za Voduška torej ni arbitraren.

Študija, ki jo objavljamo, je bila namenjena kot predavanje na 2. mednarodnem kongresu Društva za semiotične študije 1. 1979 na Dunaju. Avtorja je prehitela smrt. Linguistica z objavo izpolnjuje nalogo in se tako skuša oddolžiti spominu samoniklega jezikoslov$\mathrm{ca}, \mathrm{ki}$ je, oprt na lastno muzikalnost, $\mathrm{v}$ glasovih neprestano videl odmeve psihičnega dogajanja. 